\title{
D-MODULES, BERNSTEIN-SATO POLYNOMIALS AND F-INVARIANTS OF DIRECT SUMMANDS
}

\author{
JOSEP ÀLVAREZ MONTANER ${ }^{1}$, CRAIG HUNEKE ${ }^{2}$, AND LUIS NÚÑEZ-BETANCOURT $^{3}$
}

\begin{abstract}
We study the structure of $D$-modules over a ring $R$ which is a direct summand of a polynomial or a power series ring $S$ with coefficients over a field. We relate properties of $D$-modules over $R$ to $D$-modules over $S$. We show that the localization $R_{f}$ and the local cohomology module $H_{I}^{i}(R)$ have finite length as $D$-modules over $R$. Furthermore, we show the existence of the Bernstein-Sato polynomial for elements in $R$. In positive characteristic, we use this relation between $D$-modules over $R$ and $S$ to show that the set of $F$-jumping numbers of an ideal $I \subseteq R$ is contained in the set of $F$-jumping numbers of its extension in $S$. As a consequence, the $F$-jumping numbers of $I$ in $R$ form a discrete set of rational numbers. We also relate the Bernstein-Sato polynomial in $R$ with the $F$-thresholds and the $F$-jumping numbers in $R$.
\end{abstract}

\section{Contents}

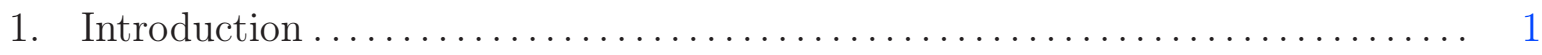

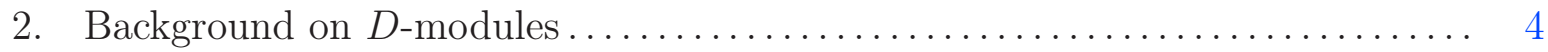

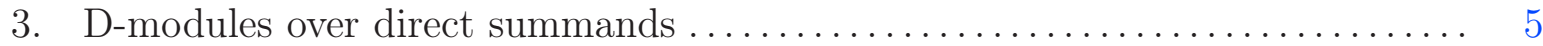

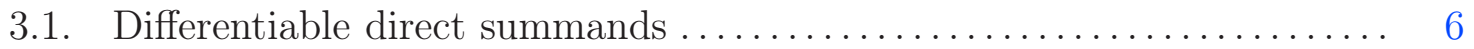

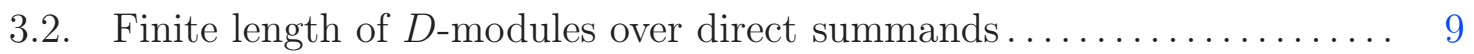

3.3. Bernstein-Sato polynomial over direct summands................. 10

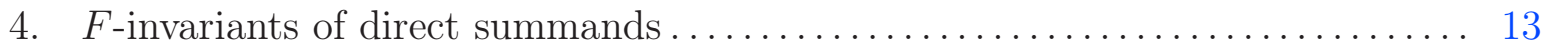

4.1. Test ideals of direct summands . . . . . . . . . . . . . . . . . . . 14

4.2. F-thresholds and Bernstein-Sato polynomials ................ 18

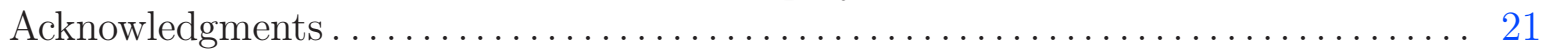

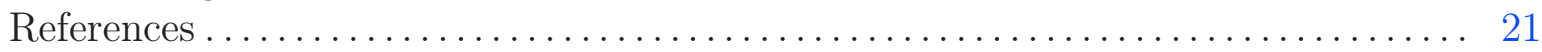

\section{INTRODUCTION}

Direct summands of polynomial rings play an important role in representation theory, combinatorial algebra, commutative algebra, and algebraic geometry. For instance, rings of invariants under a linearly reductive group actions, and affine toric rings are direct summands of polynomial rings. Rings corresponding to Grassmannian, Veronese and

2010 Mathematics Subject Classification. Primary 14F10, 13N10, 13A35, 16S32 ; Secondary 13D45, 14B05, 14M25, 13A50 .

Key words and phrases. D-modules; Bernstein-Sato polynomial; direct summands; ring of invariants; local cohomology; $F$-jumping numbers; test ideals; $F$-thresholds.

${ }^{1}$ The first author was partially supported by Generalitat de Catalunya 2014SGR-634 project and Spanish Ministerio de Economía y Competitividad MTM2015-69135-P.

${ }^{2}$ The second author was partially supported by the NSF Grant 1460638.

${ }^{3}$ The third author was partially supported by the NSF Grant 1502282 . 
Segre varieties also belong to this family of rings. If one assumes that the field has characteristic zero, then the ring associated to the $n \times m$ matrices of rank at most $t$ is a direct summand of a polynomial ring. Since the Hochster-Roberts Theorem [HR74], which states that direct summands of regular rings are Cohen-Macaulay, it has been expected that direct summands behave similarly to regular rings in certain aspects. For instance, direct summands are normal rings [HH89] with rational singularities in characteristic zero [Bou87] or strongly F-regular singularities in prime characteristic [HH89]. The main goal of this manuscript is to find structural properties of important $D$-modules over direct summands of regular rings, which resemble the situation over regular rings.

We first focus on modules over the ring of $K$-linear differential operators, $D_{R \mid K}$, where $K$ is a field, and $R$ is a direct summand of a regular $K$-algebra $S$. Significant research has been focused on the finite generation of $D_{R \mid K}$ for rings of invariants [Kan77, LS95a, Lev81, Tra06, Sch95, MVdB98, LS95b, PR05], Grassmann varieties [Tra10], and toric affine algebras [Mus87, Jon94, Mus94, ST01, ST04, ST09]. In addition, the simplicity of $D_{R \mid K}$ as a ring and the simplicity of $R$ as $D_{R \mid K^{-}}$module have also been a topic of interest [SVdB97, Smi95, Tra00]. Unfortunately, the rings of differential operators are not always finitely generated for singular rings, even in characteristic zero [BGG72]. Instead of focusing on the ring $D_{R \mid K}$, we switch gears and study the structure of an important class of $D_{R \mid K}$-modules.

We study the structure of localization and local cohomology modules over direct summands. For this we recall their finiteness properties over regular rings. Suppose that $S$ is either $K\left[x_{1}, \ldots, x_{n}\right]$ or $K\left[\left[x_{1}, \ldots, x_{n}\right]\right]$. If $K$ has characteristic zero, then the ring of $K$-linear differential operators, $D_{S \mid K}$, equals the $S$-algebra generated by the partial derivatives, $S\left\langle\frac{\partial}{\partial_{x_{1}}}, \ldots, \frac{\partial}{\partial_{x_{n}}}\right\rangle$. In particular, $D_{S \mid K}$ is a left and right Noetherian ring. Furthermore, every localization $S_{f}$, and therefore every local cohomology module $H_{I}^{i}(S)$, has finite length as $D_{S \mid K}$-module. If $K$ has prime characteristic, $D_{S \mid K}$ is no longer Noetherian. However, $S_{f}$ and $H_{I}^{i}(S)$ still have finite length as $D_{S \mid K}$-modules. These finiteness properties have a significant impact on the study of local cohomology over regular rings [Lyu93, Lyu97]. Our first result recovers these properties for direct summands.

Theorem A (see Theorem 3.8). Let $K$ be a field and $S$ be either $K\left[x_{1}, \ldots, x_{n}\right]$ or $K\left[\left[x_{1}, \ldots, x_{n}\right]\right]$, and $R$ be a $K$-subalgebra. Suppose that $R$ is a direct summand of $S$. Then $R_{f}$ and $H_{I}^{i}(R)$ have finite length as $D_{R \mid K}$-modules for every $f \in R$ and any ideal $I \subseteq R$. Furthermore,

$$
\lambda_{D_{R \mid K}}\left(R_{f}\right) \leq \lambda_{D_{S \mid K}}\left(S_{f}\right) \text { and } \lambda_{D_{R \mid K}}\left(H_{I}^{i}(R)\right) \leq \lambda_{D_{S \mid K}}\left(H_{I S}^{i}(S)\right) .
$$

The previous theorem holds for a larger class of modules (see Theorem 3.10), which includes $H_{I_{1}}^{i_{1}}\left(\cdots\left(H_{I_{\ell}}^{i_{\ell}}(R)\right) \cdots\right)$. Lyubeznik introduced a category of $D_{R \mid K}$-modules [Lyu00a, Lyu00b], denoted by $C(R, K)$, to compensate for the lack of the notion of holonomic $D$ modules in prime and mixed characteristic at the time. The category $C(R, K)$ resembles the class of holonomic modules in the sense that both consist of $D$-modules of finite length in equal characteristic.

Since its introduction [Ber72, SS72], the Bernstein-Sato polynomial $b_{f}(s)$ has been an important invariant of a hypersurface in characteristic zero. In fact, $b_{f}(s)$ is an important object in the study of singularities. For instance, it relates to the theory of vanishing cycles [Del71], $V$-filtrations and monodromies [Mal83, Kas83], jumping numbers of multiplier 
ideals [Kol97, ELSV04, BS05], and zeta functions [DL92]. In our second main result, we develop the theory of the Bernstein-Sato polynomial over a direct summand. To the best of our knowledge, this is one of the first efforts to extend the theory of the Bernstein-Sato polynomial for singular rings (see [HM16] for an study of Bernstein-Sato polynomials of ideals in a normal toric ring).

Theorem B (see Theorem 3.14). Let $K$ be a field of characteristic zero, let $S$ be either $K\left[x_{1}, \ldots, x_{n}\right]$ or $K\left[\left[x_{1}, \ldots, x_{n}\right]\right]$, and let $R$ be a $K$-subalgebra. Suppose that $R$ is a direct summand of $S$. Then for every element $f \in R \backslash\{0\}$, there exists $\delta(s) \in D_{R \mid K}[s]$ and $b(s) \in \mathbb{Q}[s]$ such that

$$
\delta(t) \cdot f^{t+1}=b(t) f^{t}
$$

for every $t \in \mathbb{Z}$. As a consequence, $R_{f}$ is a cyclic $D_{R \mid K}$-module.

Under the hypothesis of the previous theorem, we call the Bernstein-Sato polynomial of $f$ in $R, b_{f}^{R}(s)$, the monic polynomial of smallest degree satisfying the Equation 1.0.1 for some $\delta(s)$. As a consequence of the proof of Theorem B, the roots of $b_{f}^{R}(s)$ consists of negative rational numbers when $R$ is a direct summand of a polynomial ring. We hope that $b_{f}^{R}(s)$ relates to other invariants that measure singularities of hypersurfaces in direct summands.

In Remark 3.18, we show how different versions of the Bernstein-Sato theory can be extended to direct summands, which include the one given by Sabbah [Sab87] and the one given by Budur, Mustaţă, and Saito [BMS06a]. Very recently, Hsiao and Matusevish extended and studied Bernstein-Sato polynomials associated to ideals in a normal toric ring [HM16]. We note that not every $K$-algebra has Bernstein-Sato polynomials (see Example 3.19).

We point out that it was previously known that if $R$ is an affine toric ring and $I \subseteq R$ is a monomial ideal, then $H_{I}^{i}(R)$ has finite length as $D_{R \mid K}$ module [Hsi12]. In this case, it was also shown that if $f$ is a monomial, then $R_{f}$ is a cyclic $D_{R \mid K}$-module [Hsi12]. Even for toric rings, Theorems $\mathrm{A}$ and $\mathrm{B}$ recover and extend these results, as the monomial hypothesis is not longer needed.

In the second part of the paper we shift to positive characteristic to study the ring of differential operators, $D_{R}$. We show that $R_{f}$ is generated by $\frac{1}{f}$ as $D_{R}$-module for direct summands of regular rings (see Proposition 4.4). We use this fact and the ideas behind the proof of Theorems A and B to study the $F$-jumping numbers of $R$. These invariants are used to measure singularities in prime characteristic. Specifically, the $F$-jumping numbers are the values where the generalized test ideals change [HY03]. The test ideals can be seen as an analogue, in prime characteristic, of multiplier ideals [Smi97, Smi00, HY03]. In particular, the $F$-pure threshold, which is the first $F$-jumping number, serves as the analogue of the log-canonical threshold [HY03, MTW05]. We recall that multiplier ideals are defined using resolution of singularities (see [Laz04]). In contrast, test ideals are defined in terms of tight closure theory [HH90, HH94a, HH94b, HY03] so, a priori, it is not clear that $F$-jumping numbers form a discrete set of rational numbers. Since the introduction of the test ideals [HY03], intense research has been devoted around this question [BMS08, BMS09, Sch11, BSTZ10, ST14b, CEMS]. Efforts have also been dedicated to compute these invariants, mainly over regular rings [Her14, ST14b, Her15, HNBWZ16]. Our main result in this direction is that the set of the $F$-jumping numbers of $R$ is a subset of $F$-jumping numbers of $S$. 
Theorem C (see Theorem 4.12 and Corollary 4.13). Let $S$ be a regular F-finite domain, and $R$ be an $F$-finite ring. Suppose that $R \subseteq S$ and that $R$ is a direct summand of $S$. Let $I \subseteq R$ denote an ideal. Then, the set F-jumping numbers of $I$ in $R$ is a subset of the set $F$-jumping numbers of IS in $S$. In particular, the set F-jumping numbers of I in $R$ is formed by rational numbers and has no accumulation points. As a consequence, the $F$-pure threshold of $I$ is a rational number.

The previous result is relevant when the extension $R \rightarrow S$ is not finite, since the finite case follows from the work of Schwede and Tucker [ST14a].

Theorem $\mathrm{C}$ gives a set of candidates for the $F$-jumping numbers in $R$, which could potentially give algorithms to compute the invariants for direct summands (see Remark 4.14).

Motivated by the work of Mustaţă, Takagi and Watanabe [MTW05] for smooth complex varieties, we relate our notion of Bernstein-Sato polynomial to invariants in positive characteristic, namely the $F$-thresholds and the $F$-jumping numbers. These invariants are equal for regular rings [BMS08]; however, they may differ for singular rings. Suppose that $R$ is a direct summand of a polynomial ring over $\mathbb{Q}$, and $f \in R$. In Theorems 4.20 and 4.21 we show that the truncated base $p$-expansion of any $F$-jumping number and any $F$-threshold of the reduction of $f$ modulo $p$ is a root of the Bernstein-Sato polynomial $b_{f}(s)$ modulo $p$. These type of results have been used to recover roots of the Bernstein-Sato polynomials via methods in prime characteristic in the regular setting (see [BMS06b]).

\section{BACKGROUND ON D-MODUleS}

In this section we briefly recall the basics on the theory of rings of differential operators as introduced by Grothendieck [Gro67, §16.8].

Let $R$ be a Noetherian ring. The ring of differential operators of $R$ is the subring $D_{R} \subseteq \operatorname{Hom}_{\mathbb{Z}}(R, R)$ whose elements are defined inductively as follows: the differential operators of order zero are defined by the multiplication by elements of $R$, i.e. $D_{R}^{0} \cong R$. We say that $\delta \in \operatorname{Hom}_{\mathbb{Z}}(R, R)$ is an operator of order less than or equal to $m$ if $[\delta, r]=\delta r-r \delta$ is an operator of order less than or equal to $m-1$. We have a filtration $D_{R}^{0} \subseteq D_{R}^{1} \subseteq \cdots$ given by the order and the ring of differential operators is defined as

$$
D_{R}=\bigcup_{m \in \mathbb{N}} D_{R}^{m}
$$

If $A \subseteq R$ is a subring, we denote $D_{R \mid A} \subseteq D_{R}$ the subring of differential operators that are $A$-linear. In particular $D_{R}=D_{R \mid \mathbb{Z}}$. If $R$ contains a field $K$ of characteristic $p>0$,

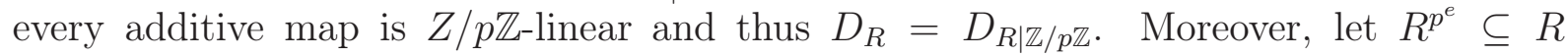
be the subring consisting of $p^{e}$ powers of elements of $R$ and set $D_{R}^{(e)}:=\operatorname{Hom}_{R^{p}}(R, R)$. In this case, $D_{R \mid K} \subseteq D_{R} \subseteq \bigcup_{e \in \mathbb{N}} D_{R}^{(e)}$. If $K$ is a perfect field, then $D_{R \mid K}=D_{R}$ and $D_{R}=\bigcup_{e \in \mathbb{N}} D_{R}^{(e)}$ when $R$ is $F$-finite (see [Smi87, Theorem 2.7] and [Yek92, Theorem 1.4.9]).

Example 2.1. Let $R$ be either the polynomial ring $A\left[x_{1}, \ldots, x_{n}\right]$ or the formal power series ring $A\left[\left[x_{1}, \ldots, x_{n}\right]\right]$ with coefficients in a ring $A$. The ring of $A$-linear differential operators is:

$$
D_{R \mid A}=R\left\langle\frac{1}{t !} \frac{d^{t}}{d x_{i}^{t}} \mid i=1, \ldots, n ; t \in \mathbb{N}\right\rangle,
$$


that is, the free $R$-module generated by the differential operators $\frac{1}{t !} \frac{d^{t}}{d x_{i}^{t}}$. Furthermore, if $A=K$ is a field of characteristic zero, we have

$$
D_{R \mid K}=R\left\langle\frac{d}{d x_{1}}, \ldots, \frac{d}{d x_{n}}\right\rangle \text {. }
$$

Example 2.2. Let $S=K\left[x_{1}, \ldots, x_{n}\right]$ be a polynomial ring over a field $K$ of characteristic zero and, given an ideal $I \subseteq S$, set $R=S / I$. Then, using the results about differential operator of quotient rings of polynomials [MR01, Theorem 5.13], the ring of $K$-linear differential operators of $R$ is given by

$$
D_{R \mid K}:=\frac{\left\{\delta \in D_{S \mid K} \mid \delta(I) \subseteq I\right\}}{I D_{S \mid K}}
$$

It is clear that $R$ is a $D_{R \mid A}$ module. Several of the results presented in this manuscript concern to the $D_{R \mid A}$-module structure of the localization, $R_{f}$ at an element $f \in R$, and the local cohomology modules $H_{I}^{i}(R)$ associated to an ideal $I \subseteq R$. For this reason, we recall a few properties and definitions regarding these objects.

- Localization: Let $M$ be a $D_{R \mid A^{-}}$module and $f \in R$. Then $M_{f}$ is also a $D_{R \mid A}$-module, where the action of a differential operator $\delta \in D_{R \mid A}$ on $\frac{v}{f^{t}} \in M_{f}$ is defined inductively as follows: If $\delta \in D_{R \mid A}^{0}$ has order zero then $\delta \cdot \frac{v}{f^{t}}=\frac{\delta \cdot v}{f^{t}}$. Now, suppose that the action of every element in $D_{R \mid A}^{n}$ has been defined. Let $\delta \in D_{R \mid A}^{n+1}$. Then,

$$
\delta \cdot \frac{v}{f^{t}}=\frac{\delta \cdot v-\left[\delta, f^{t}\right] \cdot \frac{v}{f^{t}}}{f^{t}},
$$

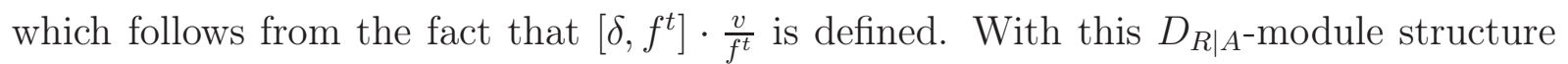
on $M_{f}$, the localization map $M \rightarrow M_{f}$ is a morphism of $D_{R \mid A}$-modules.

- Local cohomology modules: Let $I \subseteq R$ be an ideal generated by a sequence of elements $\underline{f}=f_{1}, \ldots, f_{\ell} \in R$, and let $M$ be any $R$-module. The Čech complex of $M$ with respect to $\underline{f}$ is defined by

$$
\check{\mathrm{C}}^{\bullet}(\underline{f} ; M): \quad 0 \rightarrow M \rightarrow \bigoplus_{i} M_{f_{i}} \rightarrow \bigoplus_{i, j} M_{f_{i} f_{j}} \rightarrow \cdots \rightarrow M_{f_{1} \cdots f_{\ell}} \rightarrow 0,
$$

where the maps on every summand are localization maps up to a sign. The local cohomology of $M$ with support on $I$ is defined by

$$
H_{I}^{i}(M)=H^{i}\left(\check{C}^{\bullet}(\underline{f} ; M)\right) .
$$

One may check that it is independent of the set of generators of $I$. It follows from this construction that every local cohomology module over a $D_{R \mid A^{-}}$module is again a $D_{R \mid A^{-}}$ module.

\section{D-MODUles OVER DIRECT SUMmands}

Let $A \subseteq R \subseteq S$ be an extension of Noetherian rings such that $R$ is a direct summand of $S$ with splitting morphism $\beta: S \rightarrow R$. Our first aim in this section is to relate differential operators on $S$ to differential operators on $R$ using the splitting $\beta$. Then we introduce $D_{R \mid A}$-modules that are direct summands of $D_{S \mid A}$-modules, for which the differential structure is compatible. This idea is used to obtain properties of the localizations 
$R_{f}$ and the local cohomology modules $H_{I}^{i}(R)$ as a $D_{R \mid A}$-module from the properties of $S_{f}$ and $H_{I S}^{i}(S)$ as a $D_{S \mid A}$-module. The same techniques are used in order to develop a theory of Bernstein-Sato polynomials, even if $R$ is not regular.

3.1. Differentiable direct summands. Let $A \subseteq R \subseteq S$ be Noetherian rings such that $R$ is a direct summand of $S$ with splitting morphism $\beta: S \rightarrow R$. We start relating differential operators in $D_{S \mid A}$ to differential operators in $D_{R \mid A}$ using the splitting $\beta$. We first show that the order of a differential operator cannot increase when we compose it with the splitting $\beta$. This idea has been used for rings of invariants over a group action [Sch95]. Namely, there exists a $\operatorname{map}^{1} D_{S \mid K}^{G} \rightarrow D_{S^{G} \mid K}$. However, this map may not be surjective [Sch95, Example 5.7]. It is injective for many classes of groups, in particular for finite groups, but, to the best of our knowledge, the injectivity of this map is not known in general. For the study of local cohomology modules of $S^{G}$ in characteristic zero as $D_{S \mid K}^{G}$-module, we refer to [Put16].

We point that the following lemma was implicit in Smith's proof of the statement: $S$ is a simple $D_{S \mid \mathbb{F}_{p}}$-module, then $R$ is also $D_{R \mid \mathbb{F}_{p}}$ is also simple [Smi95, Proposition 3.1]. We include this proof for the sake of completeness.

Lemma 3.1. Let $A \subseteq R \subseteq S$ be three Noetherian rings. Let $\iota: R \rightarrow S$ denote the inclusion and let $\beta: S \rightarrow R$ be any $R$-linear morphism. Then, for every $\delta \in D_{S \mid A}^{n}$, we have that $\tilde{\delta}:=\beta \circ \delta_{\left.\right|_{R}} \in D_{R \mid A}^{n}$.

Proof. To avoid heavy notation, we also write $\delta$ for $\delta_{\mid R}$ We first note that $\tilde{\delta} \in \operatorname{Hom}_{A}(R, R)$. We proceed by induction on the order $n$ of the differential operator. If $n=0$, there exists $g \in S$ such that $\delta(w)=g w$ for every $w \in S$. Then,

$$
\tilde{\delta}(v)=\beta \circ \delta(v)=\beta(\delta(v))=\beta(g v)=v \beta(g)=\beta(g) v .
$$

for every $v \in R$. We now assume the claim for $n$. Let $\delta \in D_{S \mid A}^{n+1}$, and $f \in R$. Then,

$$
\begin{aligned}
{[\tilde{\delta}, f](v) } & =\tilde{\delta}(f v)-f \tilde{\delta}(v) \\
& =\beta \circ \delta(f v)-f(\beta \circ \delta(v)) \\
& =\beta(\delta((f v))-\beta(f \delta(v)) \\
& =\beta(\delta((f v)-f \delta(v)) \\
& =\beta([\delta, f](v)) \\
& =\widetilde{[\delta, f]}(v)
\end{aligned}
$$

for all $v \in R$. Then, $[\tilde{\delta}, f]=\widetilde{[\delta, f]} \in D_{R \mid A}^{n}$ by induction hypothesis. Then, $\tilde{\delta} \in D_{S \mid A}^{n+1}$.

We now introduce $D_{R \mid A}$-modules that are direct summands of $D_{S \mid A}$-modules, for which the differential structure is compatible.

Definition 3.2. Let $A \subseteq R \subseteq S$ let be three Noetherian rings. Suppose that the inclusion $R \subseteq S$ has a splitting $\beta: S \rightarrow R$. We say that a $D_{R \mid A}$-module $M$, is a differential direct summand of the $D_{S \mid A}$-module $N$ compatible with $\beta$, if $M \subseteq N$ and we have a splitting $\theta: N \rightarrow M$ of $R$-modules such that

$$
\theta(\delta \cdot v)=\left(\beta \circ \delta_{\left.\right|_{R}}\right) \cdot v .
$$

\footnotetext{
${ }^{1} D_{S \mid K}^{G}$ denotes the ring of equivariant differential operators over the field $K$.
} 
for every $v \in M$ and $\delta \in D_{S \mid A}$. In this case, we say that $\theta$ is a differentiable splitting map compatible with $\beta$.

Lemma 3.3. Let $A \subseteq R \subseteq S$ be three Noetherian rings. Suppose that the inclusion $R \subseteq S$ has a splitting $\beta: S \rightarrow R$. Let $M \subseteq N$ be differentiable direct summand compatible with $\beta$. Let $V, W \subseteq M$ be $D_{R \mid A}$-submodules of $M$. If $D_{S \mid A} V=D_{S \mid A} W$, then $V=W$.

Proof. We denote by $\theta: N \rightarrow M$ a differential splitting, and $\beta \circ \delta_{\left.\right|_{R}}$ by $\widetilde{\delta}$. Let $v \in V$. Since $D_{S \mid A} V=D_{S \mid A} W$, there exists $\delta_{1}, \ldots, \delta_{\ell} \in D_{S \mid A}$ and $w_{1}, \ldots, w_{\ell} \in W$ such that $v=\delta_{1} w_{1}+\ldots+\delta_{\ell} w_{\ell}$. We apply $\theta$ to both sides to obtain

$$
v=\theta(v)=\theta\left(\delta_{1} w_{1}+\ldots+\delta_{\ell} w_{\ell}\right)=\theta\left(\delta_{1} w_{1}\right)+\ldots+\theta\left(\delta_{\ell} w_{\ell}\right)=\widetilde{\delta}_{1} w_{1}+\ldots+\widetilde{\delta}_{\ell} w_{\ell},
$$

where the last step follows from the fact that $\theta$ is a differential splitting compatible with $\beta$. Then, $v \in D_{R \mid A} W=W$. We conclude that $V \subseteq W$. Likewise, $W \subseteq V$, which concludes the proof.

The main result of this subsection is the following upper bound for the length, as $D$-module, of a differential direct summand.

Proposition 3.4. Let $A \subseteq R \subseteq S$ let be three Noetherian rings. Suppose that the inclusion $R \subseteq S$ has a splitting $\beta: S \rightarrow R$. Let $M \subseteq N$ be differentiable direct summands compatible with $\beta$. Then, $\lambda_{D_{R \mid A}}(M) \leq \lambda_{D_{S \mid A}}(N)$.

Proof. We note that if $N$ does not have finite length as $D_{S \mid A}$-modules the claim is clear. We assume that $\lambda_{D_{S \mid A}}(N)$ is finite, and proceed by contradiction. Let $\ell=\lambda_{D_{S \mid A}}(N)$. Let $0 \subsetneq V_{1} \subsetneq \ldots \subsetneq V_{\ell+1}$ be a strictly increasing chain of $D_{R \mid A}$-submodules of $M$. By Lemma $3.30 \subsetneq D_{S \mid A} V_{1} \subsetneq \ldots \subsetneq D_{S \mid A} V_{\ell+1}$ is a strictly increasing chain of $D_{S \mid A}$-submodules of $N$. Then, $\ell+1 \leq \lambda_{D_{S \mid A}}(N)$, which is a contradiction.

Definition 3.5. Let $A \subseteq R \subseteq S$ let be three Noetherian rings. Suppose that the inclusion $R \subseteq S$ has a splitting $\beta: S \rightarrow R$. Given two differentiable direct summands $M_{1} \subseteq N_{1}$ and $M_{2} \subseteq N_{2}$ with differentiable splittings $\theta_{1}: N_{1} \rightarrow M_{1}$ and $\theta_{2}: N_{2} \rightarrow M_{2}$, we say that a map $\phi: M_{1} \rightarrow M_{2}$ is a morphism of differential direct summands if $\phi \in \operatorname{Hom}_{D_{S \mid A}}\left(N_{1}, N_{2}\right)$, $\phi\left(M_{1}\right) \subseteq M_{2}, \phi_{\left.\right|_{M_{1}}} \in \operatorname{Hom}_{D_{R \mid A}}\left(M_{1}, M_{2}\right)$, and the following diagram

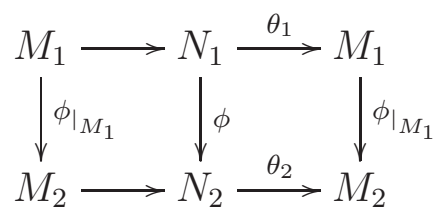

commutes. For the sake of notation, we often write $\phi$ for $\phi_{\left.\right|_{N_{1}}}$.

Proposition 3.6. Let $A \subseteq R \subseteq S$ let be three Noetherian rings. Suppose that the inclusion $R \subseteq S$ has a splitting $\beta: S \rightarrow R$. Let $M$ be a $D_{R \mid A}$-module and $N$ be a $D_{S \mid A}$-module. If $M$ is a differential direct summand of $N$, then $M_{f}$ is a differential direct summand of $N_{f}$ for every element of $f \in R$. Furthermore, the localization maps give a morphism of differential direct summands.

Proof. Let $\theta: N \rightarrow M$ be a differential splitting compatible with $\beta$. For the sake of notation, we use the same symbols for induced maps in the localization. We now show that the map $M_{f} \rightarrow N_{f}$ induced by $\theta$ is a differential splitting compatible with $\beta$. 
Let $\widetilde{\delta}$ denote the differential operator $\beta \circ \delta_{\left.\right|_{R}}$. We start showing that $\theta\left(\delta \cdot \frac{v}{f^{n}}\right)=\widetilde{\delta} \cdot \frac{v}{f^{n}}$ for every element $v \in M$ and $\delta \in D_{S \mid A}$, by induction on the order of $\delta$. If $\delta$ has order zero we have, using the action of $\delta$ as given in Section 2, that

$$
\theta\left(\delta \cdot \frac{v}{f^{n}}\right)=\theta\left(\frac{\delta \cdot v}{f^{n}}\right)=\frac{1}{f^{n}} \theta(\delta \cdot v)=\frac{1}{f^{n}} \theta(\delta \cdot v)=\frac{1}{f^{n}}(\widetilde{\delta} \cdot v),
$$

where the last equality follows because $\theta: M \rightarrow N$ is a differentiable splitting. Then,

$$
\theta\left(\delta \cdot \frac{v}{f^{n}}\right)=\frac{1}{f^{n}}(\widetilde{\delta} \cdot v)=\frac{\widetilde{\delta} \cdot v}{f^{n}}=\widetilde{\delta} \cdot \frac{v}{f^{n}} .
$$

We now assume our claim for differential operators of order equal to or less than $n$, and suppose that $\delta$ has order $n+1$.

$$
\begin{aligned}
\theta\left(\delta \cdot \frac{v}{f^{t}}\right) & =\theta\left(\frac{\delta \cdot v-\left[\delta, f^{n}\right] \cdot \frac{v}{f^{t}}}{f^{t}}\right) \\
& =\theta\left(\frac{\delta \cdot v}{f^{t}}\right)-\theta\left(\frac{\left[\delta, f^{n}\right] \cdot \frac{v}{f^{t}}}{f^{t}}\right) \\
& =\frac{1}{f^{t}} \theta(\delta \cdot v)-\theta\left(\frac{\left[\delta, f^{n}\right] \cdot \frac{v}{f^{t}}}{f^{t}}\right) \\
& =\frac{1}{f^{t}} \widetilde{\delta} \cdot v-\theta\left(\frac{\left[\delta, f^{n}\right] \cdot \frac{v}{f^{t}}}{f^{t}}\right) \text { because } \theta: M \rightarrow N \text { is a differentiable spliting } \\
& =\frac{1}{f^{t}} \widetilde{\delta} \cdot v-\frac{\widetilde{\left[\delta, f^{n}\right]} \cdot \frac{v}{f^{t}}}{f^{t}} \text { by induction hypothesis as }\left[\delta, f^{n}\right] \in D_{A}^{n}(S) ; \\
& =\frac{\widetilde{\delta} \cdot v-\widetilde{\left[\delta, f^{n}\right]} \cdot \frac{v}{f^{t}}}{\frac{f^{t}}{\left[\delta, f^{n}\right]} \cdot \frac{v}{f^{t}}} \\
& =\frac{\widetilde{\delta} \cdot v-f^{t}}{\widetilde{\delta} \cdot v-\left[\widetilde{\delta}, f^{n}\right] \cdot \frac{v}{f^{t}}} \text { as a consequence of the proof of Lemma } 3.1 ; \\
& =\frac{\widetilde{\delta} \cdot \frac{v}{f^{t}}}{}
\end{aligned}
$$

To verify that the localization map $M \rightarrow M_{f}$ induces a map of differential direct summands, we note that all the conditions in Definition 3.5 are satisfied by the usual properties of localization maps, and the fact that localization maps are $D$-module morphisms.

Lemma 3.7. Let $A \subseteq R \subseteq S$ let be three Noetherian rings. Suppose that the inclusion $R \subseteq S$ has a splitting $\beta: S \rightarrow R$. Let $M_{1} \subseteq N_{1}$ and $M_{2} \subseteq N_{2}$ be differentiable direct summands with differential splittings $\theta_{1}$ and $\theta_{2}$. Let $\phi: N_{1} \rightarrow N_{2}$ be a map of differentiable direct summands. Then, $\operatorname{Ker}\left(\phi_{\left.\right|_{M_{1}}}\right) \subseteq \operatorname{Ker}(\phi), \operatorname{Im}\left(\phi_{\left.\right|_{M_{1}}}\right) \subseteq \operatorname{Im}\left(\phi_{\left.\right|_{M_{1}}}\right)$ and $\operatorname{Coker}\left(\phi_{\left.\right|_{M_{1}}}\right) \subseteq \operatorname{Coker}(\phi)$ are also differentiable direct summands. Furthermore, the inclusion and projection maps are morphism of differentiable direct summands. 
Proof. Since $\phi$ is a map of $D_{S \mid A}$ modules and $\phi_{\left.\right|_{M_{1}}}$ is also a map of $D_{R \mid A}$-modules, we note that the induced maps on kernel, images and cokernel are also maps of $D_{S \mid A}$ and $D_{R \mid A}$-modules respectively. The rest follows from the fact that the diagram

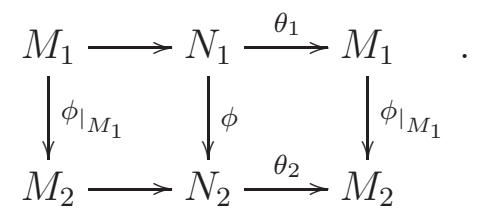

commutes, and that $\theta_{1}, \theta_{2}$ are differential splittings.

3.2. Finite length of $D$-modules over direct summands. Let $K$ be a field and $S$ be either $K\left[x_{1}, \ldots, x_{n}\right]$ or $K\left[\left[x_{1}, \ldots, x_{n}\right]\right]$. One of the main results in the theory of $D$ modules is that both the localizations $S_{f}$ and the local cohomology modules $H_{I}^{i}(S)$ have finite length as $D_{S \mid K}$-modules. If $K$ is a field of characteristic zero, this result follows from the fact that localizations and local cohomology modules belong to the class of holonomic $D_{S \mid K^{-}}$modules (see [Bjö79, Cou95]). These results can be extended to differentiably admissible $K$-algebras, which is a larger class or regular rings containing the rational numbers (see [MNM91, NB13]). For intance, if $(R, \mathfrak{m}, K)$ is a ramified regular ring of mixed characteristic $p>0$, then $R[1 / p]$ is a differentiably admissible algebra [NB13].

In positive characteristic, these results were proved by Lyubeznik using his theory of $F$-finite $F$-modules [Lyu97]. In fact, he showed that $S_{f}$ and $H_{I}^{i}(S)$ have finite length as $D_{S \mid K}$-modules for a larger class of Noetherian regular $K$-algebras of prime characteristic. Indeed, $F$-finite $F$-modules behave quite likely as holonomic modules in characteristic zero.

Despite the fact that we do not have a theory of holonomic or $F$-finite $F$-modules for direct summands, we are still able to prove the finite length of localization and local cohomology modules in this setting.

Theorem 3.8. Let $K$ be a field and $S$ be either $K\left[x_{1}, \ldots, x_{n}\right]$ or $K\left[\left[x_{1}, \ldots, x_{n}\right]\right]$. Let $K \subseteq R \subseteq S$ be a subring with a splitting $\beta: S \rightarrow R$. Then $R_{f}$ and $H_{I}^{i}(R)$ have finite length as $D_{R \mid K}$-modules for every $f \in R$ and any ideal $I \subseteq R$. Furthermore, $\lambda_{D_{R \mid K}}\left(R_{f}\right) \leq$ $\lambda_{D_{S \mid K}}\left(S_{f}\right)$ and $\lambda_{D_{R \mid K}}\left(H_{I}^{i}(R)\right) \leq \lambda_{D_{S \mid K}}\left(H_{I S}^{i}(S)\right)$.

Proof. Since $R \subseteq S$ is a differential splitting, we have that $R_{f} \subseteq S_{f}$ is a differential splitting for every $f \in R$ by Proposition 3.6. Then we have $\lambda_{D_{R \mid K}}\left(R_{f}\right) \leq \lambda_{D_{S \mid K}}\left(S_{f}\right)$ by Proposition 3.4. Let $I=\left(f_{1}, \ldots, f_{\ell}\right) \subseteq R$, and $\underline{f}=f_{1}, \ldots, f_{\ell}$. We note that $\left.\check{\mathrm{C}} \bullet \underline{f} ; R\right) \subseteq \check{\mathrm{C}}^{\bullet}(\underline{f} ; S)$ is a complex of differential direct summands, and so, the local cohomology, $H_{I}^{i}(\bar{R}) \subseteq$ $H_{I S}^{i}(S)$, is again a differential direct summand by Lemma 3.7. Then, $\lambda_{D_{R \mid K}}\left(H_{I}^{i}(R)\right) \leq$ $\lambda_{D_{S \mid K}}\left(H_{I S}^{i}(S)\right)$ by Proposition 3.4.

We may extend the property of finite length to other $D_{R \mid K}$-modules if we consider the category $C(R, A)$ introduced by Lyubeznik [Lyu00a, Lyu00b].

Definition 3.9. We denote by $C(R, A)$ the smallest subcategory of $D_{R \mid A \text {-modules that }}$ contains $R_{f}$ for all $f \in R$ and that is closed under subobjects, extensions and quotients.

The category $C(R, A)$ is also closed under localization and, in some sense, behaves as well as holonomic or F-finite F-modules. In particular, this category contains iterated local 
cohomology modules $H_{I_{1}}^{i_{1}} \cdots H_{I_{\ell}}^{i_{\ell}}(R)$, and more generally, Lyubeznik functors $\mathcal{T}$ defined in [Lyu93].

Theorem 3.10. Let $K$ be a field and $S$ be either $K\left[x_{1}, \ldots, x_{n}\right]$ or $K\left[\left[x_{1}, \ldots, x_{n}\right]\right]$. Let $K \subseteq R \subseteq S$ let be a subring with a splitting $\beta: S \rightarrow R$. Then, every $D_{R \mid K}$-module in $C(R, K)$ has finite length as $D_{R \mid K}$-module.

Proof. This statements follows from Theorem 3.8, since $R_{f}$ has finite length as $D_{R \mid K^{-}}$ module, and every object in $C(R, K)$ is build from localization modules with operations that preserve the property of having finite length.

From the previous theorem, we obtain as a corollary a result by the third author regarding associated primes of local cohomology.

Corollary 3.11 ([NB12]). Let $K$ be a field and $S$ be either $K\left[x_{1}, \ldots, x_{n}\right]$ or $K\left[\left[x_{1}, \ldots, x_{n}\right]\right]$. Let $K \subseteq R \subseteq S$ let be a subring with a splitting $\beta: S \rightarrow R$. Then, $\operatorname{Ass}_{R} H_{I}^{i}(R)$ is a finite set for every ideal $I \subseteq R$ and every integer $i$.

Proof. We note that a simple $D_{R \mid K}$-module, $M \neq 0$, has only one associated prime. In fact, if $Q$ is a maximal element in the set of the associated primes of $M$, then $\operatorname{Ass}_{R} H_{Q}^{0}(M)=Q$

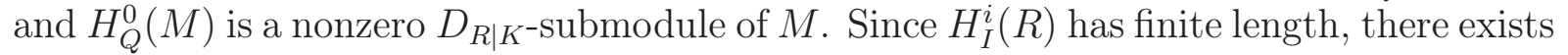
an increasing sequence of $D_{R \mid K}$-modules $0=M_{0} \subseteq M_{1} \subseteq \ldots \subseteq M_{\ell}=H_{I}^{i}(R)$ such that $M_{j+1} / M_{j}$ is a simple $D_{R \mid K}$-module. Then, $\operatorname{Ass}_{R} H_{I}^{i}(R) \subseteq \bigcup_{j=0}^{\ell} \operatorname{Ass}_{R} M_{j+1} / M_{j}$.

Remark 3.12. There are other structural properties that local cohomology modules satisfy over regular rings containing a field, which are proved using $D$-modules. For instance, local cohomology modules have finite Bass numbers and their injective dimension is bounded above by the dimension of their support. Both of these properties fail for direct summands. For instance, $R=K[a c, a d, b c, b d]$ is a direct summand of $S=K[a, b, c, d]$, $H_{(a b, a d)(R)}^{2}$ is a local cohomology module with an infinite socle [Har68], so not all Bass numbers are finite. In addition, $H_{(a b, a d)(R)}^{2}$ is a simple $D_{R \mid K}$-module [Hsi12] supported at the maximal ideal $(a b, a d, c d, b c) R$, which is not an injective $R$-module, so the injective dimension can be bigger than the dimension of the support.

3.3. Bernstein-Sato polynomial over direct summands. Let $S$ be either the polynomial ring $K\left[x_{1}, \ldots, x_{n}\right]$ or the formal power series ring $K\left[\left[x_{1}, \ldots, x_{n}\right]\right]$, where $K$ is a field of characteristic zero and $R \subseteq S$ a direct summand. We aim to extend the well-known theory of Bernstein-Sato over $S$ to the direct summand $R$. We start reviewing the basic facts.

Recall that $D_{S \mid K}=S\left\langle\frac{d}{d x_{1}}, \ldots, \frac{d}{d x_{n}}\right\rangle$ is the ring of $K$-linear differential operators of $S$. Let $D_{S \mid K}[s]$ be the polynomial ring in the indeterminate $s$ and coefficients in $D_{S \mid K}$. For any nonzero element $f \in S$, there exists a polynomial $b(s) \neq 0$ and a differential operator $\delta(s) \in D_{S \mid K}[s]$ satisfying the following functional equation:

$$
\delta(s) \cdot f \boldsymbol{f}^{\boldsymbol{s}}=b(s) \boldsymbol{f}^{\boldsymbol{s}} .
$$

This is an identity in $M\left[\boldsymbol{f}^{s}\right]:=S_{f}[s] \boldsymbol{f}^{s}$ which is the rank one free $S_{f}[s]$-module with generator $\boldsymbol{f}^{s}$ viewed as a formal symbol. After taking the specializations $M\left[\boldsymbol{f}^{\boldsymbol{s}}\right] \rightarrow S_{f}$, given by $\boldsymbol{f}^{s} \mapsto f^{t}$ for $t \in \mathbb{Z}$, we obtain the following equation

$$
\delta(t) \cdot f^{t+1}=b(t) f^{t} .
$$


The set of all polynomials $b(s)$ for which there exists an operator $\delta(s)$ such that Equation 3.3.1 holds forms an ideal of $K[s]$. The monic polynomial that generates this ideal is called the Bernstein-Sato polynomial [Ber72, SS72] and is denoted as $b_{f}(s)$.

A fundamental result states that the roots of $b_{f}(s)$ are negative rational numbers in the polynomial case $^{2}$ [Mal75, Kas77]. As a consequence, we may view $b_{f}(s) \in \mathbb{Q}[s]$.

A key point in this theory is that we can give a $D_{S \mid K}[s]$-module structure to $M\left[\boldsymbol{f}^{s}\right]$ as follows: The action of the derivatives on a general element $g \boldsymbol{f}^{\boldsymbol{s}}$ is given by

$$
\frac{d}{d x_{i}} \cdot g \boldsymbol{f}^{s}=\left(\frac{d}{d x_{i}} \cdot g+s g f^{-1} \frac{d}{d x_{i}}(f)\right) \boldsymbol{f}^{s},
$$

and the multiplication by $s$ is $D_{S \mid K}$-linear. Unfortunately, when we turn our attention to a non-regular ring $R$, we are not able to define a $D_{R \mid K}[s]$-module structure on $R_{f}[s] f^{s}$. However, it still makes sense to study the existence of an element $\delta(s) \in D_{R \mid K}[s]$ and a polynomial $b(s) \in \mathbb{Q}[s]$ that satisfy Equation 3.3.2 for every $t \in \mathbb{Z}$.

In order to make precise the setup that we introduce the following general definition that encodes all the Bernstein-Sato data.

Definition 3.13. Let $A \subseteq R$ be two Noetherian rings, and $f \in R$. We consider the $A[s]$-module,

$$
\mathcal{B}=\left\{(\delta(s), b(s)) \in D_{R \mid A}[s] \times A[s] \mid \delta(t) \cdot f^{t+1}=b(t) f^{t} \forall t \in \mathbb{Z} \text { in } R_{f}\right\} .
$$

Let $\mathcal{B}_{f}$ be the image of $\mathcal{B}$ under the projection map on the second entry. If $\mathcal{B}_{f}=0$, we say that $f$ has no Bernstein-Sato polynomial. If $\mathcal{B}_{f} \neq 0$, we call its monic generator the Bernstein-Sato polynomial of $f$, which is denoted by $b_{f}^{R_{A}}(s)$. If the rings $R$ and $A$ are clear from the context, we just write $b_{f}(s)$.

Our next result shows that Bernstein-Sato polynomials exist for a direct summand, $R \subseteq S$, as long as they exist for $S$.

Theorem 3.14. Let $A \subseteq R \subseteq S$ be three Noetherian rings. Suppose that the inclusion $R \subseteq S$ has a splitting $\beta: S \rightarrow R$. Let $f \in R$. If $b_{f}^{S_{A}}(s)$ exists, then $b_{f}^{R_{A}}(s)$ also exists. Furthermore, $b_{f}^{R_{A}}(s)$ divides $b_{f}^{S_{A}}(s)$.

Proof. Let $\delta(s) \in D_{S \mid A}[s]$ be such that $\delta(t) \cdot f^{t+1}=b(t) f^{t}$ for all $t \in \mathbb{Z}$, where the equality is taken in $S_{f}$. Since $R_{f}$ is a differential direct summand of $S_{f}$, we have that

$$
\beta \circ \delta(t)_{\left.\right|_{R}} \cdot f^{t+1}=b(t) f^{t}
$$

for every $t \in \mathbb{Z}$ in $R_{f}$. Then, $b_{f}^{S_{A}}(s)$ is a multiple of $b_{f}^{R_{A}}(s)$, since this is the monic polynomial satisfying 3.3.3.

Corollary 3.15. Let $K$ be a field and $S$ be either $K\left[x_{1}, \ldots, x_{n}\right]$ or $K\left[\left[x_{1}, \ldots, x_{n}\right]\right]$. Let $K \subseteq R \subseteq S$ be a direct summand with an splitting $\beta: S \rightarrow R$. Then the Bernstein-Sato polynomial exists for every element $f \in R$. Furthermore, in the polynomial case all the roots of $b_{f}^{R_{K}}(s)$ are negative rational numbers if $S$ is polynomial.

Proof. The Bernstein-Sato polynomial $b_{f}^{S_{K}}(s)$ exists [Ber72, SS72]. If $S$ is polynomial, $b_{f}^{S_{K}}(s)$ only has negative rational roots [Mal75, Kas77].

\footnotetext{
${ }^{2}$ Indeed, this result is also stated in the analytic case $\left(S=\mathbb{C}\left\{x_{1}, \ldots, x_{n}\right\}\right)$.
} 
Remark 3.16. Theorems 3.8, 3.10, and 3.14 can be extended to direct summands of differentiably admissible $K$-algebras, where $K$ is a field of characteristic zero (see [MNM91, NB13]). In particular, we may also consider direct summands of $S=\mathbb{C}\left\{x_{1}, \ldots, x_{n}\right\}$, and the rationality of the roots of the Bernstein-Sato polynomials also holds [Mal75, Kas77].

We now present an example that show that $b_{f}^{R}(s)$ could differ from $b_{f}^{S}(s)$. This example was kindly given to us by Jack Jeffries.

Example 3.17. Let $R=\mathbb{Q}[x u, y v]$ and $S=\mathbb{Q}[x, y, u, v]$ We note that $R=S^{G}$, where $G$ is a torus with coordinates $\lambda_{1}, \lambda_{2}$ acting by

$$
x \mapsto \lambda_{1} \lambda_{2} x, y \mapsto \lambda_{1} \lambda_{2}^{-1} y, u \mapsto \lambda_{1}^{-1} \lambda_{2}^{-1} u, v \mapsto \lambda_{1}^{-1} \lambda_{2} v .
$$

Consider $f=x u-y v$. There exists an isomorphism from $R$ to a polynomial ring in two variables, which sends $f$ to a variable. Thus, $b_{f}^{R}(s)=s+1$ and $R_{f}$ is generated by $\frac{1}{f}$ as a $D_{R \mid K^{-}}$module. In contrast, $b_{f}^{S}(s)=(s+1)(s+2)$, and so, $S_{f} \neq D_{S \mid K} \cdot \frac{1}{f}$ [Wal05, Lemma 1.3]. Furthermore, $H_{f}^{i}(R)$ is a simple $D_{R \mid K^{-}}$module, but $H_{f}^{1}(S)$ is not a simple $D_{S \mid K}$-module.

Remark 3.18. There are several variants and extensions where the theory of BernsteinSato polynomial applies. The results of Theorem 3.14 and Corollary 3.15 can be extended to these cases:

- Bernstein-Sato polynomial associated to an algebraic variety: Let $I \subseteq S$ be an ideal generated by a sequence of nonzero polynomials $f=f_{1}, \ldots, f_{\ell}$. Budur, Mustaţă and Saito [BMS06a] defined the Bernstein-Sato polynomial, $b_{I}(s)$, associated to the ideal $I$. After a convenient shifting, $b_{I}(s)$ is also an invariant of the variety $V(I)$. They proved the existence of a nonzero polynomial $b(s) \in \mathbb{Q}[s]$, and differential operators $\delta_{c}\left(s_{1}, \ldots, s_{\ell}\right) \in$ $D_{R \mid K}\left[s_{1}, \ldots, s_{\ell}\right]$ satisfying, in our setting, a functional equation

$$
\sum_{c \in \mathbb{Z}^{\ell}} \prod_{\substack{i=1, \ldots, \ell \\
c_{i}<0}} \delta_{c}\left(t_{1}, \ldots, t_{\ell}\right) \cdot\left(\begin{array}{c}
t_{i} \\
c_{i}
\end{array}\right) \prod_{i=1}^{\ell} f_{i}^{t_{i}+c_{i}}=b\left(t_{1}+\ldots+t_{r}\right) \prod_{i=1}^{\ell} f_{i}^{t_{i}}
$$

for every $t_{i} \in \mathbb{Z}$ and where the sum varies over finitely many $c=\left(c_{1}, \ldots, c_{\ell}\right) \in \mathbb{Z}^{\ell}$. We think of this as a functional equation in $S_{f_{1} \cdots f_{\ell}}$.

Let $R \subseteq S$ be a direct summand with a splitting $\beta: S \rightarrow R$ and assume that $f_{i} \in R$, $i=1, \ldots, \ell$. Then we obtain the analogue of Equation 3.3.4 for $R$ by composing the differential operators $\delta_{c}\left(t_{1}, \ldots, t_{\ell}\right)$ with the splitting $\beta$.

- Bernstein-Sato ideal associated to a family of hypersurfaces: Let $\underline{f}=f_{1}, \ldots, f_{\ell}$ be a sequence of elements in $S$. Then, there exists a nonzero polynomial $b\left(s_{1}, \ldots, s_{\ell}\right) \in$ $\mathbb{Q}\left[s_{1}, \ldots, s_{\ell}\right]$, and differential operators $\delta\left(s_{1}, \ldots, s_{\ell}\right) \in D_{R \mid K}\left[s_{1}, \ldots, s_{\ell}\right]$ satisfying, in our setting, a functional equation

$$
\delta\left(t_{1}, \ldots, t_{\ell}\right) \cdot \prod_{i=1}^{\ell} f_{i}^{t_{i}+1}=b\left(t_{1}, \ldots, t_{\ell}\right) \prod_{i=1}^{\ell} f_{i}^{t_{i}} .
$$

for every $t_{i} \in \mathbb{Z}$. The ideal in $\mathbb{Q}\left[s_{1}, \ldots, s_{\ell}\right]$ generated by these polynomials $b\left(s_{1}, \ldots, s_{\ell}\right)$ is the Bernstein-Sato ideal associated to the sequence $f$. If $R \subseteq S$ is a direct summand and $f_{i} \in R, i=1, \ldots, \ell$, we use the same compose by $\beta$ again to obtain the equation in $R$. 
We end this subsection with a example, generously suggested by Jack Jeffries, which shows that Bernstein-Sato polynomials do not exist in general.

Example 3.19. Let $R=\mathbb{C}[x, y, z] /\left(x^{3}+y^{3}+z^{3}\right)$. We consider $R$ as a graded ring where every variable has degree 1 , and we denote the $\mathbb{C}$-subspace of homogeneous elements of degree $\ell$ by $[R]_{\ell}$. We note that $D_{R \mid \mathbb{C}}$ is also a graded ring, where a differential operator has degree $\ell \in \mathbb{Z}$ if $\delta g=0$ or $\operatorname{deg}(\delta g)=\operatorname{deg}(g)+\ell$ for every homogeneous element $g \in R$. We note that this identity extends to any localization of $R$ at a homogeneous element. The $\mathbb{C}$-subspace of differential operators of degree $\ell$ is denoted by $D_{R \mid \mathbb{C}}(\ell)$. In this case, $D_{R \mid \mathbb{C}}=\bigoplus_{\ell \in \mathbb{Z}} D_{R \mid \mathbb{C}}(\ell)$. Furthermore, $D_{R \mid \mathbb{C}}(\ell)=0$ for every $\ell<0$ [BGG72, Proposition 1]. Let $f \in R$ be any element of positive degree $u$. We now show by contradiction that $f$ has no Bernstein-Sato polynomial (as in Definition 3.13). Suppose that there exists $\delta[s] \in D_{R \mid \mathbb{C}}[s]$ and $b(s) \in \mathbb{C}[s]$ such that $b(s) \neq 0$ and $\delta(t) f^{t+1}=b(t) f^{t}$ for every $t \in \mathbb{Z}$. We fix $t \in \mathbb{Z}$ such that $t>0$ and $b(t) \neq 0$. We set $\delta=\delta(t)$, and we take $\delta_{\ell} \in D_{R \mid \mathbb{C}}(\ell)$ such that $\delta=\delta_{0}+\cdots+\delta_{k}$, which is possible because there are no operators of negative degree [BGG72, Proposition 1]. Then,

$$
b(t) f^{t}=\delta f^{t+1}=\delta_{0} f^{t+1}+\cdots+\delta_{k} f^{t+1} \in \bigoplus_{\ell>(t+1) u}[R]_{\ell} .
$$

As a consequence, $t u>(t+1) u$ for every $\ell$ appearing in the equation above, because $b(t) f^{t}$ is a nonzero homogeneous element of degree $t u$. This is a contradiction because $u>0$.

Remark 3.20. We note that even in the case where $D_{R \mid K}^{1}$ has no elements of negative degree, one can find elements of negative degree in $D_{R \mid K}$. For instance, if $R=$ $\mathbb{C}[a, b, c, d] /(a d-b c) \cong \mathbb{C}[x y, x z, y u, z u]$, which is a direct summand of $S=\mathbb{C}[x, y, z, u]$ with splitting $\beta: S \rightarrow R$ defined on the monomials by $\beta\left(x^{\alpha_{1}} y^{\alpha_{2}} z^{\alpha_{3}} u^{\alpha_{4}}\right)=x^{\alpha_{1}} y^{\alpha_{2}} z^{\alpha_{3}} u^{\alpha_{4}}$ if $\alpha_{1}+\alpha_{4}=\alpha_{2}+\alpha_{3}$ and $\beta\left(x^{\alpha_{1}} y^{\alpha_{2}} z^{\alpha_{3}} u^{\alpha_{4}}\right)=0$ otherwise. Since $R$ is a graded ring with an isolated singularity, there are no elements of order 1 and negative degree [Kan77]. However, $\beta \circ \frac{\partial}{\partial x} \frac{\partial}{\partial y}$ is a differential operator on $R$ of order 2 and negative degree.

Theorem 3.14 and Example 3.19 motivate the following question.

Question 3.21. What conditions are necessary and sufficient for a $K$-algebra for the existence of the Bernstein-Sato polynomial?

\section{F-INVARIANTS OF DiRECT SUMmands}

In this section we study different invariants in positive characteristic: $F$-jumping numbers and $F$-thresholds. We point out that in a regular ring these two families of invariants are the same. However, over singular rings they usually differ, even for direct summands. For this reason, we need to study them using different approaches.

We recall that $R$ acquires an structure of $R$-module by restriction of scalars via the $e$-th iteration of the Frobenius map. $F^{e}$. We denote this module action on $R$ by $F_{*}^{e} R$. Namely, we have $r \cdot F_{*}^{e} x=F_{*}^{e}\left(r^{p^{e}} x\right) \in F_{*}^{e} R$ for $r \in R$ and $F_{*}^{e} x \in F_{*}^{e} R$. If $R$ is a reduced ring, it is a common practice to identify $F_{*}^{e} R$ with $R^{1 / p^{e}}$, via the isomorphism of $R$-modules $F_{*}^{e} r \mapsto r^{1 / p^{e}}$.

Throughout this section we consider rings containing a field of positive characteristic that are $F$-finite, that is, rings such that $F_{*}^{e} R$ is a finitely generated $R$-module. 
4.1. Test ideals of direct summands. In this subsection we prove that the $F$-jumping numbers of an ideal $I$ of a direct summand $R$ of a regular ring $S$ are contained in the $F$-jumping numbers of the extended ideal $I S$. This result follows from comparison of rings of differential operators and Cartier morphisms between $R$ and $S$. Differential operators have been used previously to show discreteness and rationality of $F$-jumping numbers for regular rings. However, they have not been used in the singular case as far as we know.

We point out that Chiecchio, Enescu, Miller, and Schwede [CEMS] used the fact that $R$ is a direct summand of the symbolic Rees algebra $\mathcal{R}=\oplus_{t=0}^{\infty} \mathcal{O}_{X}\left(-t K_{X}\right)$, to prove discreteness and rationality of $F$-jumping numbers in certain cases, given that $\mathcal{R}$ is finitely generated as $R$-algebra. From recent results in the minimal model program [BCHM10, HM10, HX15] (see also [Kol10, Theorem 92]), one could expect that $\mathcal{R}$ is finitely generated for every strongly $F$-regular ring, in particular, for direct summands of regular rings. However, to the best of our knowledge, this question is still open. Furthermore, this question relates to the longstanding problem of weakly $F$-regular versus strongly $F$-regular [CEMS]. We point out that our strategy differs from theirs by using differential operators over a singular ring.

Definition 4.1. Let $R$ be an $F$-finite Noetherian ring.

- We say that $R$ if $F$-pure if the inclusion map $R \rightarrow F_{*}^{e} R$ splits for all $e>0$.

- Assume that $R$ is a domain. We say that $R$ is strongly $F$-regular if for every $r \in R$ there exists $e \in \mathbb{N}$ such that the $\varphi: R \rightarrow F_{*}^{e} R$ defined by $1 \mapsto F_{*}^{e} r$ splits.

Regular rings are strongly $F$-regular. Moreover, direct summands of strongly $F$-regular rings are strongly $F$-regular by a result of [HH89].

We now introduce the definition and preliminary results regarding Cartier operators and its relation with differential operators in positive characteristic.

Definition 4.2. Let $R$ be an $F$-finite ring.

- An additive map $\psi: R \rightarrow R$ is a $p^{e}$-linear map if $\psi(r f)=r^{p^{e}} \psi(f)$. Let $\mathcal{F}_{R}^{e}$ be the set of all the $p^{e}$-linear maps. Then, we have $\mathcal{F}_{R}^{e} \cong \operatorname{Hom}_{R}\left(R, F_{*}^{e} R\right)$.

- An additive map $\phi: R \rightarrow R$ is a $p^{-e}$-linear map if $\phi\left(r^{p^{e}} f\right)=r \phi(f)$. Let $\mathcal{C}_{R}^{e}$ be the set of all the $p^{-e}$-linear maps. Then, we have $\mathcal{C}_{R}^{e} \cong \operatorname{Hom}_{R}\left(F_{*}^{e} R, R\right)$.

The main examples of $p^{e}$ and $p^{-e}$-linear maps are the iterated Frobenius and Cartier morphisms. For this reason, these morphisms are usually referred as Frobenius and Cartier operators. They have a close relation with differential operators. Recall from Section 2 that for $F$-finite rings we have

$$
D_{R}=\bigcup_{e} D_{R}^{(e)}
$$

Since $R$ is reduced, we have $D_{R}^{(e)} \cong \operatorname{Hom}_{R}\left(F_{*}^{e} R, F_{*}^{e} R\right)$. It is natural to consider the pairing $\mathcal{C}_{R}^{e} \otimes_{R} \mathcal{F}_{R}^{e} \longrightarrow D_{R}^{(e)}$ sending $\phi \otimes \psi$ to its composition $\psi \circ \phi$. If $R$ is a regular ring, the previous map is an isomorphism of Abelian groups, that is $\mathcal{C}_{R}^{e} \otimes_{R} \mathcal{F}_{R}^{e} \cong D_{R}^{(e)}$. One concludes, roughly speaking, that it is equivalent to consider differential operators or just Cartier operators.

In general, for singular rings, we do not have such an isomorphism. However, in the $F$-pure case we make an easy observation that relates differential operators with Cartier maps. This relation is the heart of Lemma 4.10, which is a key part of our strategy towards Theorem C. 
Remark 4.3. Let $R$ be an $F$-pure $F$-finite ring. If $\gamma: F_{*}^{e} R \rightarrow R$ is a splitting, the map $\Psi: \operatorname{Hom}_{R}\left(F_{*}^{e} R, F_{*}^{e} R\right) \rightarrow \operatorname{Hom}_{R}\left(F_{*}^{e} R, R\right)$ defined by $\delta \mapsto \gamma \circ \delta$ is surjective. Then, the corresponding map $D_{R}^{(e)} \rightarrow \mathcal{C}_{R}^{e}$ is also surjective.

Let $S$ be a regular ring. The so-called Frobenius descent, that follows from the isomorphism $\mathcal{C}_{S}^{e} \otimes_{S} \mathcal{F}_{S}^{e} \cong D_{S}^{(e)}$ and Morita equivalence, is a key ingredient to show that $S_{f}=D_{S} \cdot \frac{1}{f}$ [ĀMBL05, Theorem 1.1]. This is an important result regarding the $D$-module structure of the localization $S_{f}$ at an element $f \in S$. This result was extended by Takagi and Takahashi [TT08, Corollary 2.10] to the case of graded rings with finite F-representation type. This class of rings include graded direct summands. In our first result in this section, we prove that this occurs for all direct summands of $F$-finite regular domains.

Theorem 4.4. Let $S$ be a regular F-finite domain. Let $R \subseteq S$ be an extension of Noetherian rings such that $R$ a direct summand of $S$. Then $R_{f}$ is generated by $\frac{1}{f}$ as $D_{R}$-module.

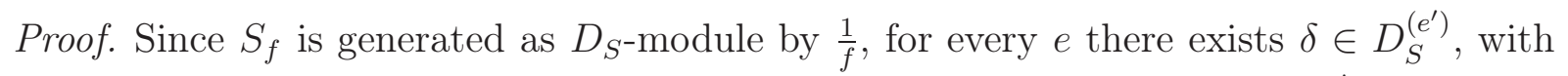
$e^{\prime} \geq e$, such that $\delta\left(\frac{1}{f}\right)=\frac{1}{f^{p^{e}}}$. Then, by multiplying this equation by $f^{p^{e^{\prime}}}$, we obtain $\delta\left(f^{p^{e^{\prime}}-1}\right)=f^{p^{e^{\prime}}-p^{e}}$.

Let $\beta: S \rightarrow R$ denote a splitting. Let $\widetilde{\delta}=\beta \circ \delta$. We note that $\widetilde{\delta} \in D_{R}^{\left(e^{\prime}\right)}$. In addition, $\widetilde{\delta}\left(f^{p^{e^{\prime}}-1}\right)=f^{p^{e^{\prime}}-p^{e}}$. Hence, $\widetilde{\delta}\left(\frac{1}{f}\right)=\frac{1}{f^{p^{e}}}$. We conclude that $R_{f}$ is generated as $D_{R^{-m o d u l e}}$ by $\frac{1}{f}$.

Test ideals have been a fundamental tool in the theory of tight closure developed by Hochster and the second author [HH90, HH94a, HH94a]. Hara and Yoshida [HY03] extended the theory to include test ideals $\tau_{R}\left(I^{\lambda}\right)$ associated to pairs $\left(R, I^{\lambda}\right)$ where $I \subseteq R$ is an ideal and $\lambda \in \mathbb{R}$ is a parameter. A new approach to test ideals ${ }^{3}$ by means of Cartier operators was given by Blickle, Mustaţă and Smith [BMS08, BMS09] in the case that $R$ is a regular ring. Their approach has been extremely useful in order to extended the theory of test ideals to non-regular rings. We refer to [Sch11, BB11, Bli13] for a more general setting.

Definition 4.5. Suppose that $R$ is a strongly $F$-regular ring. Let $I \subseteq R$ be an ideal, and $\lambda \in \mathbb{R}_{>0}$. The test ideal ${ }^{4}$ of the pair $(I, \lambda)$ is defined by

$$
\tau_{R}\left(I^{\lambda}\right)=\bigcup_{e \in \mathbb{N}} \mathcal{C}_{R}^{e} I^{\left\lceil p^{e} \lambda\right\rceil}
$$

We note that the chain of ideals $\left\{\mathcal{C}_{R}^{e} I^{\left\lceil p^{e} \lambda\right\rceil}\right\}$ is increasing [TT08, Proposition 4.4], and so, $\tau_{R}\left(I^{\lambda}\right)=\mathcal{C}_{R}^{e} I^{\left\lceil p^{e} \lambda\right\rceil}$ for $e \gg 0$.

We now summarize basic well-known properties of test ideals. In the case of strongly $F$-regular rings we refer to [TT08, Lemma 4.5]. For the general case of $F$-finite rings, we refer [Bli13, Proposition ].

Proposition 4.6. Let $R$ be an F-finite ring, $I, J \subseteq R$ ideals, and $\lambda, \lambda^{\prime} \in \mathbb{R}_{>0}$. Then, (1) If $I \subseteq J$, then $\tau_{R}\left(I^{\lambda}\right) \subseteq \tau_{R}\left(J^{\lambda}\right)$.

\footnotetext{
${ }^{3}$ Indeed, they describe the generalization to pairs of the big test ideal considered in [HH94a].

${ }^{4}$ Precisely, this are the big test ideals. This ideals are equal to the original test ideals for graded rings [LS99] and Gorenstein rings [LS01].
} 
(2) If $\lambda<\lambda^{\prime}$, then $\tau_{R}\left(I^{\lambda^{\prime}}\right) \subseteq \tau_{R}\left(I^{\lambda}\right)$.

(3) There exists $\epsilon>0$, such that $\tau_{R}\left(I^{\lambda}\right)=\tau_{R}\left(I^{\lambda^{\prime}}\right)$, if $\lambda^{\prime} \in[\lambda, \lambda+\epsilon)$.

Therefore, every ideal $I \subseteq R$ is associated to a family of test ideals $\tau_{R}\left(I^{\lambda}\right)$ parameterized by real numbers $\lambda \in \mathbb{R}_{>0}$. Indeed, they form a nested chain of ideals. The real numbers where the test ideals change are called F-jumping numbers. We now make this precise,

Definition 4.7. Let $R$ be an $F$-finite ring and let $I \subseteq R$ be an ideal. A real number $\lambda$ is an $F$-jumping number of $I$ if

for every $\epsilon>0$.

$$
\tau_{R}\left(I^{\lambda}\right) \neq \tau_{R}\left(I^{\lambda-\epsilon}\right)
$$

Remark 4.8. Let $R$ be a strongly $F$-regular $F$-finite ring of characteristic $p>0$, and $I \subseteq R$. If $R$ is either a local ring or a graded $K$ algebra with $I$ homogeneous, then the $F$-pure threshold of $I$ is defined in terms of maps $R \rightarrow F_{*}^{e} R$ that split [TW04, Definition 2.1]. Specifically,

$$
\operatorname{fpt}(I):=\sup \left\{\lambda \mid \forall e \gg 0 \exists f \in I^{\left\lfloor\left(p^{e}-1\right) \lambda\right\rfloor} \text { such that } 1 \mapsto F_{*}^{e} f \text { splits }\right\} .
$$

Then, $\operatorname{fpt}(I)$ is the first $F$-jumping number of $I$.

From now on we consider extensions of Noetherian rings $R \subseteq S$ such that $R$ is a direct summand of $S$. Our aim is to relate test ideals of an ideal in $R$ with the test ideal of its extension to $S$. The first result in this direction shows that the $F$-pure threshold of an ideal of $R$ is greater or equal than the $F$-pure threshold of its extension in $S$.

Proposition 4.9. Let $S$ be a regular $F$-finite domain. Let $R \subseteq S$ be an extension of Noetherian rings such that $R$ is a direct summand of $S$. Then, $\tau_{S}\left((I S)^{\lambda}\right) \cap R \subseteq \tau_{R}\left(I^{\lambda}\right)$ for every ideal $I \subseteq R$ and $\lambda \in \mathbb{R}_{>0}$. In particular, $\operatorname{fpt}_{S}(I S) \leq \operatorname{fpt}_{R}(I)$.

Proof. Let $f \in \tau_{S}\left((I S)^{\lambda}\right) \cap R$. Then, there exists $e \in \mathbb{N}$ such that $f \in \mathcal{C}_{S}^{e}(I S)^{\left\lceil p^{e} \lambda\right\rceil}$. Then, there exist $g_{1}, \ldots, g_{\ell} \in I^{\left\lceil p^{e} \lambda\right\rceil}$ and $\phi_{1}, \ldots, \phi_{\ell} \in C_{e}^{S}$ such that $\phi_{1}\left(g_{1}\right)+\ldots+\phi_{\ell}\left(g_{\ell}\right)=f$.

Let $\beta: S \rightarrow R$ denote a splitting. Then, $\beta \circ \phi_{\left.i\right|_{R}} \in \mathcal{C}_{R}^{e}$. Thus, $\beta\left(\phi_{i}\left(g_{i}\right)\right) \in \mathcal{C}_{R}^{e} I^{\left\lceil p^{e} \lambda\right\rceil} \subseteq$ $\tau_{R}\left(I^{\lambda}\right)$. Then,

$$
\begin{aligned}
f & =\phi_{1}\left(g_{1}\right)+\ldots+\phi_{\ell}\left(g_{\ell}\right) ; \\
& =\beta\left(\phi_{1}\left(g_{1}\right)+\ldots+\phi_{\ell}\left(g_{\ell}\right)\right) ; \\
& =\beta\left(\phi_{1}\left(g_{1}\right)\right)+\ldots+\beta\left(\phi_{\ell}\left(g_{\ell}\right)\right) .
\end{aligned}
$$

Hence, $f \in \mathcal{C}_{R}^{e}\left(I^{\left\lceil p^{e} \lambda\right\rceil}\right) \subseteq \tau_{R}\left(I^{\lambda}\right)$.

The following results are a key part of our strategy towards Theorem C.

Lemma 4.10. Let $R$ be an F-pure F-finite ring, and $I, J \subseteq R$ ideals. If $D_{R}^{(e)} I=D_{R}^{(e)} J$, then $\mathcal{C}_{R}^{e} I=\mathcal{C}_{R}^{e} J$.

Proof. We have that

$$
\begin{aligned}
\mathcal{C}_{R}^{e} I & =\operatorname{Hom}_{R}\left(F_{*}^{e} R, R\right) \cdot F_{*}^{e} I \text { by Definition } 4.2 \\
& =\gamma\left(\operatorname{Hom}_{R}\left(F_{*}^{e} R, F_{*}^{e} R\right) \cdot F_{*}^{e} I\right) \text { by Remark } 4.3 \\
& =\gamma\left(\operatorname{Hom}_{R}\left(F_{*}^{e} R, F_{*}^{e} R\right) \cdot F_{*}^{e} J\right) \text { by Definition } 4.2 \text { and } D_{R}^{(e)} I=D_{R}^{(e)} J ; \\
& =\operatorname{Hom}_{R}\left(F_{*}^{e} R, R\right) \cdot F_{*}^{e} J \text { by Remark } 4.3 \\
& =\mathcal{C}_{R}^{e} J \text { by Definition } 4.2
\end{aligned}
$$


Lemma 4.11. Let $R \subseteq S$ be an extension of Noetherian rings such that $R$ is a direct summand of $S$. Let $I \subseteq R$ denote an ideal. If $\mathcal{C}_{S}^{e}(I S)^{r}=\mathcal{C}_{S}^{e}(I S)^{t}$ for $r, t \in \mathbb{N}$, then $\mathcal{C}_{R}^{e} I^{r}=\mathcal{C}_{R}^{e} I^{t}$

Proof. We assume without loss of generality that $r \leq t$. We have $\mathcal{C}_{R}^{e} I^{t} \subseteq \mathcal{C}_{R}^{e} I^{r}$, thus we focus on the other containment. Since $\mathcal{C}_{S}^{e}(I S)^{r}=\mathcal{C}_{S}^{e}(I S)^{t}$, we have that

$$
D_{S}^{(e)}(I S)^{r}=\left(\mathcal{C}_{S}^{e}(I S)^{r}\right)^{\left[p^{e}\right]}=\left(\mathcal{C}_{S}^{e}(I S)^{t}\right)^{\left[p^{e}\right]}=D_{S}^{(e)}(I S)^{t} .
$$

Then, $I^{r} \subseteq D_{S}^{(e)}(I S)^{t}$. Let $f \in I^{r}$. Then, there exists $\phi_{1}, \ldots, \phi_{\ell} \in D_{S}^{(e)}$ and $g_{1}, \ldots, g_{\ell} \in I^{t}$ such that $f=\phi_{1}\left(g_{1}\right)+\ldots+\phi_{\ell}\left(g_{\ell}\right)$. Let $\beta: S \rightarrow R$ denote a splitting. Then, $\beta \circ \phi_{\left.i\right|_{R}} \in D_{R}^{(e)}$ and $\beta\left(\phi_{i}\left(g_{i}\right)\right) \in D_{R}^{(e)} I^{t}$. It follows that $f \in D_{R}^{(e)}\left(I^{t}\right)$ since

$$
\begin{aligned}
f & =\phi_{1}\left(g_{1}\right)+\ldots+\phi_{\ell}\left(g_{\ell}\right) \\
& =\beta\left(\phi_{1}\left(g_{1}\right)+\ldots+\phi_{\ell}\left(g_{\ell}\right)\right) \\
& =\beta\left(\phi_{1}\left(g_{1}\right)+\ldots+\beta\left(\phi_{\ell}\left(g_{\ell}\right)\right.\right. \\
& =\beta\left(\phi_{1}\left(g_{1}\right)\right)+\ldots+\beta\left(\phi_{\ell}\left(g_{\ell}\right)\right) .
\end{aligned}
$$

Therefore, $I^{r} \subseteq D_{R}^{(e)} I^{t}$. Hence, $D_{R}^{(e)} I^{r} \subseteq D_{R}^{(e)}\left(D_{R}^{(e)} I^{t}\right)=D_{R}^{(e)} I^{t}$. We conclude that $D_{R}^{(e)} I^{r}=D_{R}^{(e)} I^{t}$, and so, $\mathcal{C}_{R}^{e} I^{r}=\mathcal{C}_{R}^{e} I^{t}$ by Lemma 4.10 .

We are now ready to prove the main result in this section.

Theorem 4.12. Let $S$ be a regular F-finite domain. Let $R \subseteq S$ be an extension of Noetherian rings such that $R$ is a direct summand of $S$. Let $I \subseteq R$ denote an ideal, and $\lambda_{1}, \lambda_{2} \in \mathbb{R}_{>0}$. If $\tau_{S}\left((I S)^{\lambda_{1}}\right)=\tau_{S}\left((I S)^{\lambda_{2}}\right)$, then $\tau_{R}\left(I^{\lambda_{1}}\right)=\tau_{R}\left(I^{\lambda_{2}}\right)$.

Proof. There exists $t \in \mathbb{N}$ such that for $e>N, \tau_{S}\left((I S)^{\lambda_{1}}\right)=\mathcal{C}_{S}^{e}(I S)^{\left\lceil p^{e} \lambda_{1}\right\rceil}$ and $\tau_{S}\left((I S)^{\lambda_{2}}\right)=$ $\mathcal{C}_{S}^{e}(I S)^{\left\lceil p^{e} \lambda_{2}\right\rceil}$. Then, $\mathcal{C}_{S}^{e}(I S)^{\left\lceil p^{e} \lambda_{1}\right\rceil}=\mathcal{C}_{S}^{e}(I S)^{\left\lceil p^{e} \lambda_{2}\right\rceil}$ for $e \geq t$. By Lemma 4.11, we have that $\mathcal{C}_{R}^{e} I^{\left\lceil p^{e} \lambda_{1}\right\rceil}=\mathcal{C}_{R}^{e} I^{\left\lceil p^{e} \lambda_{2}\right\rceil}$ for $e \geq t$. Then,

$$
\tau_{R}\left(I^{\lambda_{1}}\right)=\bigcup_{e \in \mathbb{N}} \mathcal{C}_{R}^{e} I^{\left\lceil p^{e} \lambda_{1}\right\rceil}=\bigcup_{e \geq t} \mathcal{C}_{R}^{e} I^{\left\lceil p^{e} \lambda_{1}\right\rceil}=\bigcup_{e \geq t} \mathcal{C}_{R}^{e} I^{\left\lceil p^{e} \lambda_{2}\right\rceil}=\bigcup_{e \in \mathbb{N}} \mathcal{C}_{R}^{e} I^{\left\lceil p^{e} \lambda_{2}\right\rceil}=\tau_{R}\left(I^{\lambda_{2}}\right) .
$$

Corollary 4.13. Let $S$ be a regular $F$-finite domain. Let $R \subseteq S$ be an extension of $F$-finite Noetherian rings such that $R$ is a direct summand of $S$. Let $I \subseteq R$ denote an ideal. Then, the set of F-jumping numbers of $I$ in $R$ is a subset of the set of F-jumping numbers of IS in $S$. In particular, the set of F-jumping numbers of $I$ in $R$ is formed by rational numbers and has no accumulation points.

Proof. The first claim follows immediately from the definition of $F$-jumping numbers and Theorem 4.12. The second claim follows from the fact that the set of $F$-jumping numbers consits of rational numbers and it has no accumulation points for $F$-finite regular domains [ST14b, Theorem B].

Remark 4.14. The previous result gives candidates to compute the $F$-jumping numbers of $I$. Furthermore, we obtain an algorithm to find the test ideals and $F$-jumping numbers of $I$ given that we know the corresponding objects for $I S$. 
If $\tau_{S}\left(I S^{\lambda}\right)=\mathcal{C}_{S}^{e}(I S)^{\left[p^{e} \lambda\right\rceil}$ for some $e \gg 0$, then $\tau_{R}\left(I^{\lambda}\right)=\mathcal{C}_{R}^{e} I^{\left[p^{e} \lambda\right\rceil}$. As a consequence, we can know whether a $F$-jumping number of $I S$ is also a $F$-jumping number of $I$. To do this, we need to compute first the next $F$-jumping number for $I S$, say $\lambda^{\prime}$. We now compute where the test ideal of $\tau_{S}\left(I S^{\lambda}\right)$ and $\tau_{S}\left(I S^{\lambda^{\prime}}\right)$ stabilize. We use this to compute the test ideals $\tau_{R}\left(I^{\lambda}\right)$ and $\tau_{R}\left(I^{\lambda^{\prime}}\right)$. Then, these ideals differ if and only if $\lambda$ is an $F$-jumping number for $I$ in $R$.

It is natural to ask if the conclusion of Corollary 4.13 holds in characteristic zero.

Question 4.15. Let $S=K\left[x_{1}, \ldots, x_{n}\right]$ be a polynomial ring over a field of characteristic zero. Let $R \subseteq S$ be such that $R$ a direct summand of $S$. Let $I \subseteq R$ denote an ideal. Is the set of jumping numbers for the multiplier ideals of $I$ in $R$ a subset of the set jumping numbers for the multiplier ideals of $I S$ in $S$ ?

4.2. F-thresholds and Bernstein-Sato polynomials. Mustaţă, Takagi and Watanabe introduced some asymptotic invariants associated to an ideal $\mathfrak{a}$ in an $F$-finite regular local ring $R$ [MTW05]. Roughly speaking, given any ideal $J \subseteq R$, the $F$-threshold $c^{J}(\mathfrak{a})$ measures the containment of powers of $\mathfrak{a}$ in the Frobenius powers of $J$. The set of $F$ thresholds when we vary $J$ coincides with the set of $F$-jumping numbers of $\mathfrak{a}$ [BMS08, Corollary 2.30]. Together with the second author, they extended the notion of $F$-threshold to all Noetherian rings in prime characteristic [HMTW08]. This definition is given by a limit, which they showed to exists for $F$-pure rings. This limit was recently showed to exist in full generality [DSNBP16, Theorem A]. In this subsection we extend previous relations between $F$-threshold, $F$-jumping numbers, and the Bernstein-Sato polynomial, previously known only for polynomial rings [MTW05]. For the sake of exposition, we restrict ourselves to principal ideals and to finitely generated $\mathbb{Q}$-algebras. We start by recalling this relation in the regular case. Let $S=\mathbb{Q}\left[x_{1}, \ldots, x_{n}\right]$. Let $\bar{f}$ denote the class of $f$ in $\mathbb{F}_{p}\left[x_{1}, \ldots, x_{n}\right]$, where $p$ is greater any denominator appearing in $f$. Then, for $p \gg 0$,

$$
b_{f}^{S}\left(\nu_{f}^{\mathfrak{a}}\left(p^{e}\right)\right) \equiv 0 \quad \bmod p
$$

for every ideal $\mathfrak{a} \subseteq \mathbb{F}_{p}\left[x_{1}, \ldots, x_{n}\right]$ such that $\bar{f} \in \sqrt{\mathfrak{a}}$ [MTW05, Proposition 3.11].

We start by recalling the definition of $F$-thresholds.

Definition 4.16. Let $R$ be a Noetherian rings, and $J, \mathfrak{a} \subseteq R$ be ideals such that $J \subseteq \sqrt{\mathfrak{a}}$. Let $\nu_{J}^{\mathfrak{a}}\left(p^{e}\right)=\max \left\{t \mid J^{t} \nsubseteq \mathfrak{a}^{\left[p^{e}\right]}\right\}$. We denote the $F$-threshold of $J$ with respect to $\mathfrak{a}$ by

$$
c^{\mathfrak{a}}(J)=\lim _{e \rightarrow \infty} \frac{\nu_{J}^{\mathfrak{a}}\left(p^{e}\right)}{p^{e}} .
$$

It is worth mentioning that, in the singular case, the $F$-thresholds may differ from the $F$-jumping numbers (see [HWY14, Section 6] for a few examples). For this reason, it is not clear, a priori, that $F$-thresholds are rational numbers that form a discrete set. We note that this is the case if $R$ is a direct summand of a regular ring $S$.

Proposition 4.17. Let $S$ be a regular F-finite domain. Let $R \subseteq S$ be a direct summand of $S$. Then, $c^{\mathfrak{a}}(J)$ is a rational number for every ideal $J \subseteq R$. Furthermore, the set

$$
\left\{c^{\mathfrak{a}}(J) \mid \mathfrak{a} \subseteq R \& J \subseteq \sqrt{\mathfrak{a}}\right\}
$$

has no accumulation points. 
Proof. We have that $c^{\mathfrak{a}}(J)=c^{\mathfrak{a} S}(J S)$ [HMTW08, Proposition 2.2(v)]. Every $F$-threshold, $c^{\mathfrak{a} S}(J S)$, is an $F$-jumping number for test ideals of $\mathfrak{a}$ [BMS08]. Both statements, rationality and discreteness, follows from the fact that the set of $F$-jumping numbers consists in rational numbers and it has no accumulation points for $F$-finite regular domains [ST14b, Theorem B].

We now start relating the Bernstein-Sato polynomial with $F$-thresholds for direct summands. For this we need to establish a lemma that allow us to restrict $\mathbb{Q}$-linear differential operators to $\mathbb{Z}$-linear operators.

Lemma 4.18. Let $S=\mathbb{Q}\left[x_{1}, \ldots, x_{n}\right], I \subseteq S$ be an ideal, and $R=S / I$. Let $S_{\mathbb{Z}}=$ $\mathbb{Z}\left[x_{1}, \ldots, x_{n}\right], J=I \cap S_{\mathbb{Z}}$, and $R_{\mathbb{Z}}=S_{\mathbb{Z}} / J$. Then, for every $\delta \in D_{R \mid \mathbb{Q}}$, there exists $c \in \mathbb{N}$ such that $c \delta\left(R_{\mathbb{Z}}\right) \subseteq R_{\mathbb{Z}}$. Furthermore, $c \delta \in D_{R \mid \mathbb{Z}}$.

Proof. Recall that

$$
D_{R \mid \mathbb{Q}}=\frac{\left\{\partial \in D_{S \mid \mathbb{Q}} \mid \partial(I) \subseteq I\right\}}{I D_{S \mid \mathbb{Q}}} .
$$

Given $\delta \in D_{R \mid \mathbb{Q}}$, let $\partial \in D_{S \mid \mathbb{Q}}$ be such that $\delta f=\overline{\partial f}$ for every $\bar{f} \in R$. Since

$$
D_{S \mid \mathbb{Q}}=S\left\langle\frac{d}{d x_{1}}, \ldots, \frac{d}{d x_{n}}\right\rangle
$$

there exists an integer $c$ such that

$$
c \partial \in S_{\mathbb{Z}}\left\langle\frac{d}{d x_{1}}, \ldots, \frac{d}{d x_{n}}\right\rangle .
$$

Furthermore, $c \partial\left(S_{\mathbb{Z}}\right) \subseteq S_{\mathbb{Z}}$ and $c \partial(J) \subseteq J$. It follows that $c \delta f=\overline{c \partial f} \in R_{\mathbb{Z}}$ for every $\bar{f} \in R_{\mathbb{Z}}$. Moreover, $c \delta \in D_{R \mid \mathbb{Z}}$ as $c \partial \in D_{S_{\mathbb{Z}} \mid \mathbb{Z}}$.

Remark 4.19. Let $R$ be a finitely generated $\mathbb{Z}$-algebra. Then, there is a ring morphism $D_{R \mid \mathbb{Z}} \rightarrow D_{R \otimes_{\mathbb{Z}} \mathbb{F}_{p} \mid \mathbb{F}_{p}}$, defined by $\delta \mapsto \bar{\delta}$, where $\bar{\delta} \cdot \bar{f}=\overline{\delta \cdot f}$. Furthermore, this map sends $D_{R \mid \mathbb{Z}}^{n}$ to $D_{R \otimes_{\mathbb{Z}} \mathbb{F}_{p} \mid \mathbb{F}_{p}}^{n}$. We point out that this map may not be surjective [Smi95, Pages 384-385].

We now relate the Bernstein-Sato polynomial to F-thresholds.

Theorem 4.20. Let $S=\mathbb{Q}\left[x_{1}, \ldots, x_{n}\right], I \subseteq S$ be an ideal, and $R=S / I$. Let $S_{\mathbb{Z}}=$ $\mathbb{Z}\left[x_{1}, \ldots, x_{n}\right], J=I \cap S_{\mathbb{Z}}$, and $R_{\mathbb{Z}}=S_{\mathbb{Z}} / J$. Suppose that $b_{f}^{R}(s)$ exists. Then, there exists $m \in \mathbb{N}$, depending only on $R_{\mathbb{Z}}$, such that

$$
b_{f}^{R}\left(\nu \frac{\mathfrak{a}}{f}\left(p^{e}\right)\right) \equiv 0 \quad \bmod p
$$

for every prime number $p>m$, and every ideal $\mathfrak{a} \subseteq R_{\mathbb{Z}} / p R_{\mathbb{Z}}$ such that $\bar{f} \in \sqrt{\mathfrak{a}}$. In particular, this holds when $R$ is a direct summand of a polynomial ring over $\mathbb{Q}$.

Proof. We pick a differential operator $\delta(s)=\sum_{j=1}^{m} \delta_{j} s^{j} \in D_{R \mid \mathbb{Q}}[s]$ such that

$$
\delta(t) f^{t+1}=b_{f}(t) f^{t}
$$

for every $t \in \mathbb{Z}$. By Lemma 4.18 we may clean up the coefficients of $\delta(s)$ so, without any loss of generality, we may assume that $\delta(s) \in D_{R \mid \mathbb{Z}}[s]$ as long as we pick $p>c$, where $c$ is as in Lemma 4.18. Moreover, me may multiply both sides of Equation 4.2 .1 by the least common multiple of the denominators of every coefficient of $b_{f}^{R}(s)$. We pick $p$ bigger than any of this denominators. We may also assume that it is an equation in $R_{\mathbb{Z}}$. 
Now we reduce this equation $\bmod p$. By our choice of $p, b_{f}^{R}(s)$ is not the zero polynomial in $\mathbb{F}_{p}[s]$. We have the equation

$$
\overline{\delta(t)}^{t+1}={\overline{b_{f}(t)}}^{t}
$$

for every $t \in \mathbb{N}$. We pick $p>\max n_{j}$, where $n_{j}$ denotes the order of the differential operator $\delta_{j}$. Then, the order of $\overline{\delta(t)}$ is bounded above by $p-1$, so $\overline{\delta(t)} \in D_{R}^{(1)}=\operatorname{Hom}_{R}\left(F_{*} R, F_{*} R\right)$ for every $t \in \mathbb{N}$. Let $\mathfrak{a} \subseteq R_{\mathbb{Z}} / p R_{\mathbb{Z}}$ such that $\bar{f} \in \sqrt{\mathfrak{a}}$. We set $\nu=\nu_{f}^{\mathfrak{a}}\left(p^{e}\right)$, thus we have that $\bar{f}^{\nu+1} \in \mathfrak{a}^{\left[p^{e}\right]}$ and $\overline{\delta(\nu+1)} \mathfrak{a}^{\left[p^{e}\right]} \subseteq \mathfrak{a}^{\left[p^{e}\right]}$. Then,

$$
b_{f}(\nu) \bar{f}^{\nu}=\overline{\delta(\nu)}_{\bar{f}}^{\nu+1} \in \mathfrak{a}^{\left[p^{e}\right]} .
$$

Since $\bar{f} \notin I^{\left[p^{e}\right]}$, we deduce that $b_{f}(\nu) \equiv 0 \bmod p$.

We also point out that $\nu_{f}^{\mathfrak{a}}\left(p^{e}\right)$ is the $e$-truncation of the base $p$-expansion of $c^{\mathfrak{a}}(f)$. For a similar result for $F$-pure thresholds, we refer to [Her12, Key Lemma].

Recall that the set of $F$-thresholds may differ from the set of $F$-jumping numbers for non-regular rings. Our next result shows that we still have an analogous relation between Bernstein-Sato polynomials and $F$-jumping numbers.

Theorem 4.21. Let $S=\mathbb{Q}\left[x_{1}, \ldots, x_{n}\right], I \subseteq S$ be an ideal, and $R=S / I$. Let $S_{\mathbb{Z}}=$ $\mathbb{Z}\left[x_{1}, \ldots, x_{n}\right], J=I \cap S_{\mathbb{Z}}$, and $R_{\mathbb{Z}}=S_{\mathbb{Z}} / J$. Suppose that $b_{f}^{R}(s)$ exists. Then, there exists $m \in \mathbb{N}$, depending only on $R_{\mathbb{Z}}$,

$$
b_{f}^{R}(\nu) \equiv 0 \quad \bmod p
$$

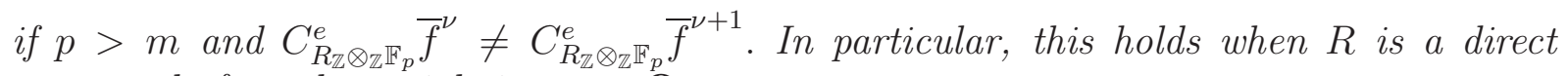
summand of a polynomial ring over $\mathbb{Q}$.

Proof. As in the proof of Theorem 4.20, for $p \gg 0$, we can assume that $\delta(s) \in D_{R \mid \mathbb{Z}}[s]$. We can assume that no denominator in any coefficient in $b_{f}^{R}(s)$ becomes zero. Then, $b_{f}^{R}(s)$ is not the zero polynomial in $\mathbb{F}_{p}[s]$. We have the equation

$$
\overline{\delta(t)}^{t+1}={\overline{b_{f}(t)}}^{t}
$$

for every $t \in \mathbb{N}$. We can also assume that $\overline{\delta(t)} \in D_{R}^{(1)}=\operatorname{Hom}_{R}\left(F_{*} R, F_{*} R\right)$ for every $t \in \mathbb{N}$. If $b_{f}(\nu) \not \equiv 0 \bmod p, \bar{f}^{\nu} \in D^{(1)} \bar{f}^{\nu+1}$. Then,

$$
D^{(e)} \bar{f}^{\nu} \subseteq D^{(e)} D^{(1)} \bar{f}^{\nu+1}=D^{(e)} \bar{f}^{\nu+1} .
$$

Since $D^{(e)} \bar{f}^{a+1} \subseteq D^{(e)} \bar{f}^{\nu}$, we conclude that $D^{(e)} \bar{f}^{\nu}=D^{(e)} \bar{f}^{\nu+1}$. Then, $C_{R_{\mathbb{Z}} \otimes_{\mathbb{Z}} \mathbb{F}_{p}} \bar{f}^{\nu}=$ $C_{R_{\mathbb{Z} \otimes_{\mathbb{Z}} \mathbb{F}_{p}}^{e}} \bar{f}^{\nu+1}$ by Remark 4.10, which contradicts our hypothesis. Hence, $b_{f}(\nu) \equiv 0 \bmod$ $p$.

Remark 4.22. Theorem 4.20 and 4.21 also hold for ideals that are not principal if we consider the Bernstein-Sato polynomial for arbitrary ideals (see Remark 3.18). These claims can be also extended for any field of characteristic zero. We focused on principal ideals over finitely generated $\mathbb{Q}$-algebras for the sake of accessibility. The experts would easily adapt the arguments in the proof to the more general setting. 


\section{ACKNOWLEDGMENTS}

We thank Jack Jeffries for suggesting Examples 3.17 and 3.19. We also thank Jen-Chieh Hsiao, Luis Narváez-Macarro and Karl Schwede for several helpful comments. The third author is grateful to Karen E. Smith for inspiring conversations.

\section{REFERENCES}

[ÀMBL05] Josep Àlvarez Montaner, Manuel Blickle, and Gennady Lyubeznik. Generators of $D$-modules in positive characteristic. Math. Res. Lett., 12(4):459-473, 2005. 15

[BB11] Manuel Blickle and Gebhard Böckle. Cartier modules: finiteness results. J. Reine Angew. Math., 661:85-123, 2011. 15

[BCHM10] Caucher Birkar, Paolo Cascini, Christopher D. Hacon, and James McKernan. Existence of minimal models for varieties of log general type. J. Amer. Math. Soc., 23(2):405-468, 2010. 14

[Ber72] I. N. Bernštel̆n. Analytic continuation of generalized functions with respect to a parameter. Funkcional. Anal. i Priložen., 6(4):26-40, 1972. 2, 11

[BGG72] I. N. Bernšter̆n, I. M. Gel'fand, and S. I. Gel'fand. Differential operators on a cubic cone. Uspehi Mat. Nauk, 27(1(163)):185-190, 1972. 2, 13

[Bjö79] Jan-Erik Björk. Rings of differential operators, volume 21 of North-Holland Mathematical Library. North-Holland Publishing Co., Amsterdam, 1979. 9

[Bli13] Manuel Blickle. Test ideals via algebras of $p^{-e}$-linear maps. J. Algebraic Geom., 22(1):49-83, 2013. 15

[BMS06a] Nero Budur, Mircea Mustaţă, and Morihiko Saito. Bernstein-Sato polynomials of arbitrary varieties. Compos. Math., 142(3):779-797, 2006. 3, 12

[BMS06b] Nero Budur, Mircea Mustaţă, and Morihiko Saito. Roots of Bernstein-Sato polynomials for monomial ideals: a positive characteristic approach. Math. Res. Lett., 13(1):125-142, 2006. 4

[BMS08] Manuel Blickle, Mircea Mustaţă, and Karen E. Smith. Discreteness and rationality of Fthresholds. Michigan Math. J., 57:43-61, 2008. Special volume in honor of Melvin Hochster. $3,4,15,18,19$

[BMS09] Manuel Blickle, Mircea Mustaţă, and Karen E. Smith. F-thresholds of hypersurfaces. Trans. Amer. Math. Soc., 361(12):6549-6565, 2009. 3, 15

[Bou87] Jean-François Boutot. Singularités rationnelles et quotients par les groupes réductifs. Invent. Math., 88(1):65-68, 1987. 2

[BS05] Nero Budur and Morihiko Saito. Multiplier ideals, $V$-filtration, and spectrum. J. Algebraic Geom., 14(2):269-282, 2005. 3

[BSTZ10] Manuel Blickle, Karl Schwede, Shunsuke Takagi, and Wenliang Zhang. Discreteness and rationality of $F$-jumping numbers on singular varieties. Math. Ann., 347(4):917-949, 2010. 3

[CEMS] Alberto Chiecchio, Florian Enescu, Lance Miller, and Karl Schwede. Test ideals in rings with finitely generated anti-canonical algebras. Journal of the Institute of Mathematics of Jussieu, to appear. 3,14

[Cou95] S. C. Coutinho. A primer of algebraic D-modules, volume 33 of London Mathematical Society Student Texts. Cambridge University Press, Cambridge, 1995. 9

[Del71] Pierre Deligne. Théorie de Hodge. I. In Actes du Congrès International des Mathématiciens (Nice, 1970), Tome 1, pages 425-430. Gauthier-Villars, Paris, 1971. 2

[DL92] J. Denef and F. Loeser. Caractéristiques d'Euler-Poincaré, fonctions zêta locales et modifications analytiques. J. Amer. Math. Soc., 5(4):705-720, 1992. 3

[DSNBP16] Alessando De Stefani, Luis Núñez-Betancourt, and Felipe Pérez. On the existence of Fthresholds and related limits. arXiv:1605.03264, 2016. 18

[ELSV04] Lawrence Ein, Robert Lazarsfeld, Karen E. Smith, and Dror Varolin. Jumping coefficients of multiplier ideals. Duke Math. J., 123(3):469-506, 2004. 3 
[Gro67] A. Grothendieck. Éléments de géométrie algébrique. IV. Étude locale des schémas et des morphismes de schémas IV. Inst. Hautes Études Sci. Publ. Math., (32):361, 1967. 4

[Har68] Robin Hartshorne. Cohomological dimension of algebraic varieties. Ann. of Math. (2), 88:403-450, 1968. 10

[Her12] Daniel J. Hernández. F-purity of hypersurfaces. Math. Res. Lett., 19(2):389-401, 2012. 20

[Her14] Daniel J. Hernández. F-pure thresholds of binomial hypersurfaces. Proc. Amer. Math. Soc., 142(7):2227-2242, 2014. 3

[Her15] Daniel J. Hernández. F-invariants of diagonal hypersurfaces. Proc. Amer. Math. Soc., 143(1):87-104, 2015. 3

[HH89] Melvin Hochster and Craig Huneke. Tight closure and strong F-regularity. Mém. Soc. Math. France (N.S.), (38):119-133, 1989. Colloque en l'honneur de Pierre Samuel (Orsay, 1987). 2,14

[HH90] Melvin Hochster and Craig Huneke. Tight closure, invariant theory, and the Briançon-Skoda theorem. J. Amer. Math. Soc., 3(1):31-116, 1990. 3, 15

[HH94a] Melvin Hochster and Craig Huneke. F-regularity, test elements, and smooth base change. Trans. Amer. Math. Soc., 346(1):1-62, 1994. 3, 15

[HH94b] Melvin Hochster and Craig Huneke. Tight closure of parameter ideals and splitting in module-finite extensions. J. Algebraic Geom., 3(4):599-670, 1994. 3

[HM10] Christopher D. Hacon and James McKernan. Existence of minimal models for varieties of log general type. II. J. Amer. Math. Soc., 23(2):469-490, 2010. 14

[HM16] Jen-Chieh Hsiao and Laura Felicia Matusevich. Bernstein-Sato polynomials on normal toric varieties. arXiv:1608.03646, 2016. 3

[HMTW08] Craig Huneke, Mircea Mustaţă, Shunsuke Takagi, and Kei-ichi Watanabe. F-thresholds, tight closure, integral closure, and multiplicity bounds. Michigan Math. J., 57:463-483, 2008. Special volume in honor of Melvin Hochster. 18, 19

[HNBWZ16] Daniel J. Hernández, Luis Núñez-Betancourt, Emily E. Witt, and Wenliang Zhang. F-Pure thresholds of homogeneous polynomials. Michigan Math. J., 65(1):57-87, 2016. 3

[HR74] Melvin Hochster and Joel L. Roberts. Rings of invariants of reductive groups acting on regular rings are Cohen-Macaulay. Advances in Math., 13:115-175, 1974. 2

[Hsi12] Jen-Chieh Hsiao. D-module structure of local cohomology modules of toric algebras. Trans. Amer. Math. Soc., 364(5):2461-2478, 2012. 3, 10

[HWY14] Daisuke Hirose, Kei-ichi Watanabe, and Ken-ichi Yoshida. F-thresholds versus $a$-invariants for standard graded toric rings. Comm. Algebra, 42(6):2704-2720, 2014. 18

[HX15] Christopher D. Hacon and Chenyang Xu. On the three dimensional minimal model program in positive characteristic. J. Amer. Math. Soc., 28(3):711-744, 2015. 14

[HY03] Nobuo Hara and Ken-Ichi Yoshida. A generalization of tight closure and multiplier ideals. Trans. Amer. Math. Soc., 355(8):3143-3174 (electronic), 2003. 3, 15

[Jon94] A. G. Jones. Rings of differential operators on toric varieties. Proc. Edinburgh Math. Soc. (2), 37(1):143-160, 1994. 2

[Kan77] Jean-Michel Kantor. Formes et opérateurs différentiels sur les espaces analytiques complexes. Bull. Soc. Math. France Mém., (53):5-80, 1977. 2, 13

[Kas83] M. Kashiwara. Vanishing cycle sheaves and holonomic systems of differential equations. In Algebraic geometry (Tokyo/Kyoto, 1982), volume 1016 of Lecture Notes in Math., pages 134-142. Springer, Berlin, 1983. 2

[Kas77] Masaki Kashiwara. $B$-functions and holonomic systems. Rationality of roots of $B$-functions. Invent. Math., 38(1):33-53, 1976/77. 11, 12

[Kol97] János Kollár. Singularities of pairs. In Algebraic geometry-Santa Cruz 1995, volume 62 of Proc. Sympos. Pure Math., pages 221-287. Amer. Math. Soc., Providence, RI, 1997. 3

[Kol10] János Kollár. Exercises in the birational geometry of algebraic varieties. In Analytic and algebraic geometry, volume 17 of IAS/Park City Math. Ser., pages 495-524. Amer. Math. Soc., Providence, RI, 2010. 14

[Laz04] Robert Lazarsfeld. Positivity in algebraic geometry. II, volume 49 of Ergebnisse der Mathematik und ihrer Grenzgebiete. 3. Folge. A Series of Modern Surveys in Mathematics [Results 
in Mathematics and Related Areas. 3rd Series. A Series of Modern Surveys in Mathematics]. Springer-Verlag, Berlin, 2004. Positivity for vector bundles, and multiplier ideals. 3

[Lev81] Thierry Levasseur. Grade des modules sur certains anneaux filtrés. Comm. Algebra, 9(15):1519-1532, 1981. 2

[LS95a] T. Levasseur and J. T. Stafford. Invariant differential operators and an homomorphism of Harish-Chandra. J. Amer. Math. Soc., 8(2):365-372, 1995. 2

[LS95b] T. Levasseur and J. T. Stafford. Invariant differential operators and an homomorphism of Harish-Chandra. J. Amer. Math. Soc., 8(2):365-372, 1995. 2

[LS99] Gennady Lyubeznik and Karen E. Smith. Strong and weak $F$-regularity are equivalent for graded rings. Amer. J. Math., 121(6):1279-1290, 1999. 15

[LS01] Gennady Lyubeznik and Karen E. Smith. On the commutation of the test ideal with localization and completion. Trans. Amer. Math. Soc., 353(8):3149-3180 (electronic), 2001. 15

[Lyu93] Gennady Lyubeznik. Finiteness properties of local cohomology modules (an application of D-modules to commutative algebra). Invent. Math., 113(1):41-55, 1993. 2, 10

[Lyu97] Gennady Lyubeznik. $F$-modules: applications to local cohomology and $D$-modules in characteristic $p>0$. J. Reine Angew. Math., 491:65-130, 1997. 2, 9

[Lyu00a] Gennady Lyubeznik. Finiteness properties of local cohomology modules: a characteristicfree approach. J. Pure Appl. Algebra, 151(1):43-50, 2000. 2, 9

[Lyu00b] Gennady Lyubeznik. Finiteness properties of local cohomology modules for regular local rings of mixed characteristic: the unramified case. Comm. Algebra, 28(12):5867-5882, 2000. Special issue in honor of Robin Hartshorne. 2, 9

[Mal75] B. Malgrange. Le polynôme de Bernstein d'une singularité isolée. In Fourier integral operators and partial differential equations (Colloq. Internat., Univ. Nice, Nice, 1974), pages 98-119. Lecture Notes in Math., Vol. 459. Springer, Berlin, 1975. 11, 12

[Mal83] B. Malgrange. Polynômes de Bernstein-Sato et cohomologie évanescente. In Analysis and topology on singular spaces, II, III (Luminy, 1981), volume 101 of Astérisque, pages 243267. Soc. Math. France, Paris, 1983. 2

[MNM91] Z. Mebkhout and L. Narváez-Macarro. La thèorie du polynôme de Bernstein-Sato pour les algèbres de Tate et de Dwork-Monsky-Washnitzer. Ann. Sci. École Norm. Sup. (4), 24(2):227-256, 1991. 9, 12

[MR01] J. C. McConnell and J. C. Robson. Noncommutative Noetherian rings, volume 30 of Graduate Studies in Mathematics. American Mathematical Society, Providence, RI, revised edition, 2001. With the cooperation of L. W. Small. 5

[MTW05] Mircea Mustaţă, Shunsuke Takagi, and Kei-ichi Watanabe. F-thresholds and Bernstein-Sato polynomials. pages 341-364, 2005. 3, 4, 18

[Mus87] Ian M. Musson. Rings of differential operators on invariant rings of tori. Trans. Amer. Math. Soc., 303(2):805-827, 1987. 2

[Mus94] Ian M. Musson. Differential operators on toric varieties. J. Pure Appl. Algebra, 95(3):303315, 1994. 2

[MVdB98] Ian M. Musson and Michel Van den Bergh. Invariants under tori of rings of differential operators and related topics. Mem. Amer. Math. Soc., 136(650):viii+85, 1998. 2

[NB12] Luis Núñez-Betancourt. Local cohomology properties of direct summands. J. Pure Appl. Algebra, 216(10):2137-2140, 2012. 10

[NB13] Luis Núñez-Betancourt. On certain rings of differentiable type and finiteness properties of local cohomology. J. Algebra, 379:1-10, 2013. 9, 12

[PR05] W. Plesken and D. Robertz. Constructing invariants for finite groups. Experiment. Math., 14(2):175-188, 2005. 2

[Put16] Tony J. Puthenpurakal. Local cohomology modules of invariant rings. Math. Proc. Cambridge Philos. Soc., 160(2):299-314, 2016. 6

[Sab87] C. Sabbah. Proximité évanescente. II. Équations fonctionnelles pour plusieurs fonctions analytiques. Compositio Math., 64(2):213-241, 1987. 3

[Sch95] Gerald W. Schwarz. Lifting differential operators from orbit spaces. Ann. Sci. École Norm. Sup. (4), 28(3):253-305, 1995. 2, 6 
[Sch11] Karl Schwede. Test ideals in non-QQQ-Gorenstein rings. Trans. Amer. Math. Soc., 363(11):5925-5941, 2011. 3, 15

[Smi87] S. P. Smith. The global homological dimension of the ring of differential operators on a nonsingular variety over a field of positive characteristic. J. Algebra, 107(1):98-105, 1987. 4

[Smi95] Karen E. Smith. The D-module structure of F-split rings. Math. Res. Lett., 2(4):377-386, 1995. 2, 6, 19

[Smi97] Karen E. Smith. F-rational rings have rational singularities. Amer. J. Math., 119(1):159180, 1997. 3

[Smi00] Karen E. Smith. The multiplier ideal is a universal test ideal. Comm. Algebra, 28(12):59155929, 2000. Special issue in honor of Robin Hartshorne. 3

[SS72] Mikio Sato and Takuro Shintani. On zeta functions associated with prehomogeneous vector spaces. Proc. Nat. Acad. Sci. U.S.A., 69:1081-1082, 1972. 2, 11

[ST01] Mutsumi Saito and William N. Traves. Differential algebras on semigroup algebras. In Symbolic computation: solving equations in algebra, geometry, and engineering (South Hadley, MA, 2000), volume 286 of Contemp. Math., pages 207-226. Amer. Math. Soc., Providence, RI, 2001. 2

[ST04] Mutsumi Saito and William N. Traves. Finite generation of rings of differential operators of semigroup algebras. J. Algebra, 278(1):76-103, 2004. 2

[ST09] Mutsumi Saito and Ken Takahashi. Noetherian properties of rings of differential operators of affine semigroup algebras. Osaka J. Math., 46(2):529-556, 2009. 2

[ST14a] Karl Schwede and Kevin Tucker. On the behavior of test ideals under finite morphisms. $J$. Algebraic Geom., 23(3):399-443, 2014. 4

[ST14b] Karl Schwede and Kevin Tucker. Test ideals of non-principal ideals: computations, jumping numbers, alterations and division theorems. J. Math. Pures Appl. (9), 102(5):891-929, 2014. $3,17,19$

[SVdB97] Karen E. Smith and Michel Van den Bergh. Simplicity of rings of differential operators in prime characteristic. Proc. London Math. Soc. (3), 75(1):32-62, 1997. 2

[Tra00] William N. Traves. Tight closure and differential simplicity. J. Algebra, 228(2):457-476, 2000. 2

[Tra06] William N. Traves. Differential operators on orbifolds. J. Symbolic Comput., 41(12):12951308, 2006. 2

[Tra10] Will Traves. Differential operators on Grassmann varieties. In Symmetry and spaces, volume 278 of Progr. Math., pages 197-207. Birkhäuser Boston, Inc., Boston, MA, 2010. 2

[TT08] Shunsuke Takagi and Ryo Takahashi. $D$-modules over rings with finite $F$-representation type. Math. Res. Lett., 15(3):563-581, 2008. 15

[TW04] Shunsuke Takagi and Kei-ichi Watanabe. On F-pure thresholds. J. Algebra, 282(1):278-297, 2004. 16

[Wal05] Uli Walther. Bernstein-Sato polynomial versus cohomology of the Milnor fiber for generic hyperplane arrangements. Compos. Math., 141(1):121-145, 2005. 12

[Yek92] Amnon Yekutieli. An explicit construction of the Grothendieck residue complex. Astérisque, (208):127, 1992. With an appendix by Pramathanath Sastry. 4

Deptartament de Matemàtiques, Universitat Politècnica de Catalunya, Av. Diagonal 647, Barcelona 08028, Spain

E-mail address: Josep.Alvarez@upc.edu

Department of Mathematics, University of Virginia, Charlottesville, VA 22904-4135, USA

E-mail address: huneke@virginia.edu

Centro de Investigación en Matemáticas, Guanajuato, Gto., México

E-mail address: luisnub@cimat.mx 